\title{
On $\mathrm{Q}_{6}$ flavor symmetry and the breaking of $\mu \leftrightarrow \tau$ symmetry
}

\author{
Juan Carlos Gómez-Izquierdo, $, 2,3$, 用F. Gonzalez-Canales,, , 5, † and M. Mondragón ${ }^{2}$, 用 \\ 1 Tecnologico de Monterrey, Campus Estado de Mexico, Atizapan \\ de Zaragoza, Estado de Mexico, Apartado Postal 52926, Mexico. \\ ${ }^{2}$ Instituto de Física, Universidad Nacional Autónoma de México, \\ Apdo. Postal 20-364, CDMX 01000, México. \\ ${ }^{3}$ Departamento de Física, Centro de Investigación y de Estudios Avanzados del I. P. N., \\ Apdo. Post. 14-740, 07000, Ciudad de México, México. \\ 4 Fac. de Cs. de la Electrónica, Benemérita Universidad Autónoma de Puebla, Apdo. Postal 542, \\ Puebla, Pue. 72000, México. \\ ${ }^{5}$ Centro Internacional de Física Fudamental, Benemérita Universidad Autónoma de Puebla.
}

\begin{abstract}
In the simplest version of a $\mathbf{Q}_{6}$ flavored supersymmetric model, we analyze the leptonic masses and mixings in the framework of a soft breaking of the $\mu \leftrightarrow \tau$ symmetry. This breaking is controlled by the inequality $m_{e \tau} \neq m_{e \mu}$ in the effective neutrino mass. As a consequence of this breaking, the reactor and atmospheric angle are deviate from $0^{\circ}$ and $45^{\circ}$, respectively. Such deviations can be enhanced or suppressed by the CP parities in the Majorana phases, so that an analytic study is carried out to remark their importance to constrain the free parameters that accommodate the mixing angles. The normal hierarchy is completely discarded in this model, the inverted hierarchy is less favored than the degenerate one where the reactor and atmospheric angles are in good agreement with the experimental data. Additionally, the model predicts defined regions for the effective neutrino mass decay, the neutrino mass scale and the sum of the neutrino mass in the inverted and degenerate mass spectrum. Thus, this model may be testable by future experiments that focus in neutrinoless double beta decay.
\end{abstract}

PACS numbers:

\section{INTRODUCTION}

In the last two decades the neutrino oscillation experiments have provided a large amount of evidence in favor of the massive neutrinos and leptonic flavor mixings. In the theoretical framework of three active neutrinos we need only six independent parameters in order to characterize the so-called ordinary neutrino oscillations. These parameters

\footnotetext{
*Electronic address: jcgizquierdo1979@gmail.com

${ }^{\dagger}$ Electronic address: felixfcoglz@gmail.com

${ }^{\ddagger}$ Electronic address: myriam@fisica.unam.mx
} 
are: the difference of the squared neutrino masses, the flavor mixing angles and the "Dirac-like" CP violation phase factor [1]. The numerical values for the squared neutrino masses and flavor mixing angles obtained from a global fit to the current experimental data on neutrino oscillations, at Best Fit Point (BFP) $\pm 1 \sigma$ and $3 \sigma$ ranges, are $[2$ :

$$
\begin{aligned}
& \Delta m_{21}^{2}\left(10^{-5} \mathrm{eV}^{2}\right)=7.60_{-0.18}^{+0.19}, 7.11-8.18, \quad\left|\Delta m_{31}^{2}\right|\left(10^{-3} \mathrm{eV}^{2}\right)=\left\{\begin{array}{l}
2.48_{-0.07}^{+0.05}, 2.30-2.65 \\
2.38_{-0.06}^{+0.05}, 2.20-2.54
\end{array}\right. \\
& \sin ^{2} \theta_{12} / 10^{-1}=3.23 \pm 0.16,2.78-3.75, \\
& \sin ^{2} \theta_{13} / 10^{-2}=\left\{\begin{array}{l}
2.26 \pm 0.12,1.90-2.62 \\
2.29 \pm 0.12,1.93-2.65
\end{array}\right.
\end{aligned}
$$

The upper and lower rows are for a normal and inverted hierarchy of the neutrino mass spectrum, respectively. Currently, the experimental determination of the above parameters is in a precision age. However, this is not the case for the "Dirac-like" CP violation phase in the leptonic sector, since the T2K experiment only give us hints of an approximately maximal $\mathrm{CP}$ violation phase, $\delta_{\mathrm{CP}} \sim-\pi / 2\left[\underline{3}\right.$. Then, the $\delta_{\mathrm{CP}}$ phase from a global fit to the current experimental data on neutrino oscillations, at $\mathrm{BFP} \pm 1 \sigma$ and $3 \sigma$ level, is [2]:

$$
\delta_{\mathrm{CP}} /^{\circ}=\left\{\begin{array}{c}
254_{-72}^{+99}, 0-360 \\
266 \pm 56,0-360
\end{array} .\right.
$$

The above experimental evidence was enough to show that neutrinos have a tiny mass, whereby it was very easy to conclude that there is physics beyond the Standard Model (SM). Now we have the dilemma how far beyond the SM we have to go and under what arguments this extension is based. In this work we consider the current experimental data on neutrino oscillations that were mentioned above. So, from these data we obtain that the magnitude of the leptonic mixing matrix elements have the following values at $3 \sigma$

- Normal Hierarchy (NH)

$$
\left(\begin{array}{lll}
0.780-0.842 & 0.520-0.607 & 0.137-0.162 \\
0.207-0.555 & 0.395-0.714 & 0.618-0.794 \\
0.226-0.566 & 0.420-0.731 & 0.590-0.772
\end{array}\right)
$$

- Inverted Hierarchy (IH)

$$
\left(\begin{array}{ccc}
0.779-0.842 & 0.520-0.607 & 0.139-0.163 \\
0.207-0.554 & 0.397-0.710 & 0.626-0.792 \\
0.229-0.566 & 0.426-0.729 & 0.592-0.765
\end{array}\right)
$$

From the leptonic mixing matrices given in the Eqs. (3) and 44 we obtain for the second and third row the remarkable result

$$
\left|U_{\mu 1}\right| \approx\left|U_{\tau 1}\right|, \quad\left|U_{\mu 2}\right| \approx\left|U_{\tau 2}\right|, \quad\left|U_{\mu 3}\right| \approx\left|U_{\tau 3}\right|
$$

This means that the leptonic mixing matrix has in an approximate way the so called $\mu \leftrightarrow \tau$ symmetry. In the model building context, the $\mu \leftrightarrow \tau$ flavor symmetry has been widely used to propose possible extensions of the SM. In these extensions, the $\mu \leftrightarrow \tau$ flavor symmetry can be defined in two different ways: 
i) the $\mu \leftrightarrow \tau$ permutation symmetry [4] where the neutrino mass term is unchanged under the transformations $\nu_{e} \rightarrow \nu_{e}, \nu_{\mu} \rightarrow \nu_{\tau}$ and $\nu_{\tau} \rightarrow \nu_{\mu}$

ii) the $\mu \leftrightarrow \tau$ reflection symmetry [10 12] where the neutrino mass term is unchanged under the transformations $\nu_{e} \rightarrow \nu_{e}^{c}, \nu_{\mu} \rightarrow \nu_{\tau}^{c}$ and $\nu_{\tau} \rightarrow \nu_{\mu}^{c}$, where $c$ denotes the charge conjugation.

In here we will only consider the first definition, hence in the following when we mention the $\mu \leftrightarrow \tau$ symmetry actually we mean the $\mu \leftrightarrow \tau$ permutation symmetry. Historically, theoretical physicists have proposed the $\mu \leftrightarrow \tau$ symmetry in order to reproduce the experimental data on lepton mixing angles. Namely, the $\mu \leftrightarrow \tau$ symmetry is obtained if the neutrino oscillation parameters fulfill one of the following conditions

$$
\left|U_{\mu i}\right|=\left|U_{\tau i}\right| \Longleftrightarrow\left\{\begin{array}{l}
\theta_{13}=0^{\circ} \text { and } \theta_{23}=45^{\circ} \\
\delta= \pm 90^{\circ} \text { and } \theta_{23}=45^{\circ}
\end{array}\right.
$$

The first one is ruled out by the current experimental data on neutrino oscillations, but not even the second condition is allowed at the $1 \sigma$ or $3 \sigma$ level. At present, the Long-baseline energy experiment NO $\nu \mathrm{A}$ has disfavored the exact $\mu-\tau$ symmetry, therefore some possible breakings of the $\mu \leftrightarrow \tau$ symmetry have been explored [13] 24] (for generic models see [11, 25 32]). If this symmetry is broken, it is vital to investigate the source of the breaking and the framework where this is realized.

Based on the above, we build a supersymmetric model with the $\mathbf{Q}_{6}$ symmetry group as flavor symmetry and conserved $R$-parity. In this context, as a first step we study the masses and flavor mixing in the leptonic sector, where the $\mu \leftrightarrow \tau$ symmetry is only broken in the effective neutrino mass matrix, by the difference $m_{e \tau}-m_{e \mu} \neq 0$, which deviates the atmospheric angle from $45^{\circ}$ and the reactor angle is non zero. In previous works on $\mathbf{Q}_{6}[33$, 39 , the scalar sector was extended such that three families of doublets $H_{i}^{d}$ and $H_{i}^{u}$ are needed for the mixing. Contrary other models reported in the literature, we will try to explain the contrast between the CKM and PMNS mixing matrices by assigning in a different way the quark and lepton sector under the action of the flavor symmetry, as we will see below. The quark sector will be analyzed in a future work.

\section{THE MODEL}

In our model the matter content and how it transforms under the action of the $\mathbf{Q}_{6}$ flavor symmetry is shown in TableI. As we can see, the quark and lepton sectors have been assigned in a different way under the action of the flavor symmetry; the main reason to do so is to take seriously the remarkable hierarchy in the quark masses. Although the charged lepton masses may preserve this hierarchy, in the neutrinos sector this is not clear. So the quark and Higgs

\begin{tabular}{|c|c|c|c|c|c|c|}
\hline \hline $\mathbf{Q}_{6}$ & $1_{+, 0}$ & $1_{+, 2}$ & $1_{-, 1}$ & $1_{-, 3}$ & $2_{2}$ & $2_{1}$ \\
\hline Matter & $H_{3}^{d}$ & $H_{3}^{u}, Y_{B}$ & $L_{1}, N_{1}^{c}, Q_{3}, u_{3}^{c}$ & $\ell_{1}^{c}, d_{3}^{c}$ & $L_{J}, \ell_{J}^{c}, N_{J}^{c}, Q_{I}, d_{I}^{c}, u_{I}^{c}, H_{I}^{d}$ & $H_{I}^{u}$ \\
\hline \hline
\end{tabular}

TABLE I: Matter content.

superfields $Q_{I}$ and $H_{I}^{u, d}$, with $I=1,2$, transform as doublets. In fact, for the rest of the superfields, if they have the subscript $I$, it means that they transform as doublets under the flavor group, otherwise, the superfields transform as singlets under the flavor group. This flavor structure provides hierarchical quark mass matrices that reproduce the 
CKM mixing matrix quite well 33 39. On the other hand, in the leptonic sector, the first family is assigned to any of singlets of $\mathbf{Q}_{6}$, while the other two families are assigned as elements of the flavor doublet, $L_{J}$ with $J=2,3$. In here, we would like to emphasize that such assignment is a possible route for realizing the $\mu \leftrightarrow \tau$ symmetry [7, 19, 40, 42, and the tribimaximal mixing matrix [43, 44]. In comparison to previous works on $\mathbf{Q}_{6}$ [33, 39], where the assignment $\mathbf{2} \oplus \mathbf{1}$ (the first two families in the $\mathbf{2}$ and the third one in the singlet irreducible representations) for the families of fermions and scalars was used, in this paper, we assigned differently the leptons $(\mathbf{1} \oplus \mathbf{2}$, the first family in the singlet and the second and third one in the doublet) in order to identify and subsequently break the $\mu \leftrightarrow \tau$ symmetry, as we will see later.

In this theoretical framework the superpotential has the form:

$$
\begin{aligned}
\mathbf{W}= & y_{1}^{u}\left(Q_{1} u_{2}^{c}-Q_{2} u_{1}^{c}\right) H_{3}^{u}+y_{2}^{u}\left(Q_{1} H_{2}^{u}+Q_{2} H_{1}^{u}\right) u_{3}^{c}+y_{3}^{u} Q_{3}\left(u_{1}^{c} H_{2}^{u}+u_{2}^{c} H_{1}^{u}\right)+y_{4}^{u} Q_{3} u_{3}^{c} H_{3}^{u} \\
& +y_{1}^{d}\left[Q_{1}\left(-d_{1}^{c} H_{1}^{d}+d_{2}^{c} H_{2}^{d}\right)+Q_{2}\left(d_{1}^{c} H_{2}^{d}+d_{2}^{c} H_{1}^{d}\right)\right]+y_{2}^{d}\left(Q_{1} d_{1}^{c}+Q_{2} d_{2}^{c}\right) H_{3}^{d}+y_{3}^{d} Q_{3} d_{3}^{c} H_{3}^{d} \\
& +y_{1}^{\ell} L_{1} e_{1}^{c} H_{3}^{d}+y_{2}^{\ell}\left[L_{2}\left(-e_{2}^{c} H_{1}^{d}+e_{3}^{c} H_{2}^{d}\right)+L_{3}\left(e_{2}^{c} H_{2}^{d}+e_{3}^{c} H_{1}^{d}\right)\right]+y_{3}^{\ell}\left(L_{2} e_{2}^{c}+L_{3} e_{3}^{c}\right) H_{3}^{d}+y_{1}^{D} L_{1} N_{1}^{c} H_{3}^{u} \\
& +y_{2}^{D} L_{1}\left(N_{2}^{c} H_{2}^{u}+N_{3}^{c} H_{1}^{u}\right)+y_{3}^{D}\left(L_{2} H_{2}^{u}+L_{3} H_{1}^{u}\right) N_{1}^{c}+y_{4}^{D}\left(L_{2} N_{3}^{c}-L_{3} N_{2}^{c}\right) H_{3}^{u}+y^{m} Y_{B} N_{1}^{c} N_{1}^{c} \\
& +M_{R_{2}}\left(N_{2}^{c} N_{2}^{c}+N_{3}^{c} N_{3}^{c}\right)
\end{aligned}
$$

Before going ahead we will make some important remarks. $i$ ) In order to generate the flavor invariant Majorana mass term for the right-handed neutrinos, we included one Babu-Kubo flavon $Y_{B K}$ [33. ii) From the matter content given in Table I we obtain that the $\mu$-terms in the Higgs sector are not flavor invariant, whereby these kind of terms do not appear in the Higgs sector. However, the $\mu$-terms must be present in the Higgs sector because these are essential to get the electroweak symmetry breaking. Consequently, extra gauge singlets should be included in order to build the $\mu$-terms invariant under the action of the flavor group (see [45, 46]). iii) As the main aim of this work is the implication of the $\mu \leftrightarrow \tau$ symmetry breaking in the leptonic masses and mixings, in this version of the model we assume a particular alignment in the vacuum expectation values (vev's) of the scalar fields.

\section{THE LEPTON MASSES AND FLAVOR MIXING ANGLES}

From the superpotential given in Eq. (7) the form of the Dirac fermions mass matrices in the leptonic sector is the following

$$
\mathbf{M}_{D}=\left(\begin{array}{ccc}
y_{1}^{D}\left\langle\mathbf{H}_{3}^{u}\right\rangle & y_{2}^{D}\left\langle\mathbf{H}_{2}^{u}\right\rangle & y_{2}^{D}\left\langle\mathbf{H}_{1}^{u}\right\rangle \\
y_{3}^{D}\left\langle\mathbf{H}_{2}^{u}\right\rangle & 0 & y_{4}^{D}\left\langle\mathbf{H}_{3}^{u}\right\rangle \\
y_{3}^{D}\left\langle\mathbf{H}_{1}^{u}\right\rangle & -y_{4}^{D}\left\langle\mathbf{H}_{3}^{u}\right\rangle & 0
\end{array}\right) \quad \text { and } \quad \mathbf{M}_{\ell}=\left(\begin{array}{ccc}
y_{1}^{\ell}\left\langle\mathbf{H}_{3}^{d}\right\rangle & 0 & 0 \\
0 & y_{3}^{\ell}\left\langle\mathbf{H}_{3}^{d}\right\rangle-y_{2}^{\ell}\left\langle\mathbf{H}_{1}^{d}\right\rangle & y_{2}^{\ell}\left\langle\mathbf{H}_{2}^{d}\right\rangle \\
0 & y_{2}^{\ell}\left\langle\mathbf{H}_{2}^{d}\right\rangle & y_{3}^{\ell}\left\langle\mathbf{H}_{3}^{d}\right\rangle+y_{2}^{\ell}\left\langle\mathbf{H}_{1}^{d}\right\rangle
\end{array}\right)
$$

Here, $\mathbf{M}_{D}$ is the Dirac neutrinos mass matrix, while $\mathbf{M}_{\ell}$ is the charged leptons mass matrix. In the flavor space, the Majorana right-handed (RHD) neutrino mass matrix has the diagonal form

$$
\mathbf{M}_{R}=\operatorname{diag}\left(M_{R_{1}}, M_{R_{2}}, M_{R_{2}}\right)
$$

where $M_{R_{1}}=y^{n}\left\langle Y_{B K}\right\rangle$.

As it is well known, the $\mu \leftrightarrow \tau$ symmetry in the effective neutrinos mass matrix is identified very well in the basis where the charged lepton mass matrix is diagonal. In our model, this can be accomplished by the following alignment in the scalar sector $\left\langle\mathbf{H}_{1}^{u}\right\rangle=\left\langle\mathbf{H}_{2}^{u}\right\rangle$, and $\left\langle\mathbf{H}_{2}^{d}\right\rangle=0$; at the same time such alignment allows us to reduce free parameters in the Dirac neutrino mass matrix, and as a consequence in the effective neutrino mass matrix as well, where a partial 
$\mu \leftrightarrow \tau$ symmetry is expected. Then, the mass matrices given in Eq. (8) acquire the following forms, respectively,

$$
\mathbf{M}_{D}=\left(\begin{array}{ccc}
a_{D} & b_{D} & b_{D} \\
c_{D} & 0 & d_{D} \\
c_{D} & -d_{D} & 0
\end{array}\right) \quad \text { and } \quad \mathbf{M}_{\ell}=\operatorname{diag}\left(a_{\ell}, b_{\ell}, d_{\ell}\right)
$$

where $a_{D} \equiv y_{1}^{D}\left\langle\mathbf{H}_{3}^{u}\right\rangle, b_{D} \equiv y_{2}^{D}\left\langle\mathbf{H}_{1}^{u}\right\rangle, c_{D} \equiv y_{3}^{D}\left\langle\mathbf{H}_{1}^{u}\right\rangle, d_{D} \equiv y_{4}^{D}\left\langle\mathbf{H}_{3}^{u}\right\rangle, a_{\ell} \equiv y_{1}^{\ell}\left\langle\mathbf{H}_{3}^{d}\right\rangle, b_{\ell} \equiv y_{3}^{\ell}\left\langle\mathbf{H}_{3}^{d}\right\rangle-y_{2}^{\ell}\left\langle\mathbf{H}_{1}^{d}\right\rangle$, and $d_{\ell} \equiv y_{3}^{\ell}\left\langle\mathbf{H}_{3}^{d}\right\rangle+y_{2}^{\ell}\left\langle\mathbf{H}_{1}^{d}\right\rangle$. As the mass matrix $\mathbf{M}_{\ell}$ has a diagonal shape, the physical masses for the charged leptons are $m_{e}=\left|a_{\ell}\right|, m_{\mu}=\left|b_{\ell}\right|$ and $m_{\tau}=\left|d_{\ell}\right|$. So, all information about the leptonic flavor mixing only comes from the neutrino sector.

In this theoretical framework, the active Majorana neutrino mass matrix $\mathbf{M}_{\nu}$ is obtained through the type-I seesaw mechanism [47 53, $\mathbf{M}_{\nu}=\mathbf{M}_{D} \mathbf{M}_{R}^{-1} \mathbf{M}_{D}^{\top}$, where the $\mathbf{M}_{D}$ and $\mathbf{M}_{R}$ matrices are given in Eqs. (9) and 10 . Hence, the explicit form of $\mathbf{M}_{\nu}$ is

$$
\mathbf{M}_{\nu}=\left(\begin{array}{lll}
m_{e e} & m_{e \mu} & m_{e \tau} \\
m_{e \mu} & m_{\mu \mu} & m_{\mu \tau} \\
m_{e \tau} & m_{\mu \tau} & m_{\mu \mu}
\end{array}\right)
$$

where

$$
\begin{aligned}
& m_{e e}=\frac{a_{D}^{2}}{M_{R_{1}}}+2 \frac{c_{D}^{2}}{M_{R_{2}}}, m_{e \mu}=\frac{a_{D} b_{D}}{M_{R_{1}}}-\frac{c_{D} d_{D}}{M_{R_{2}}}, m_{e \tau}=\frac{a_{D} b_{D}}{M_{R_{1}}}+\frac{c_{D} d_{D}}{M_{R_{2}}}, \\
& m_{\mu \mu}=\frac{b_{D}^{2}}{M_{R_{1}}}+\frac{d_{D}^{2}}{M_{R_{2}}}, \quad m_{\mu \tau}=\frac{b_{D}^{2}}{M_{R_{1}}} .
\end{aligned}
$$

Remarkably, in this flavored model, the $\mu-\tau$ symmetry is only broken by the entries $m_{e \mu}$ and $m_{e \tau}\left(m_{e \mu} \neq m_{e \tau}\right)$. On the contrary, in generic models, where the charged lepton mass matrix is diagonal, the $m_{\tau \tau} \neq m_{\mu \mu}$ difference also breaks the $\mu-\tau$ symmetry; this implies extra free parameters in the effective neutrino mass matrix $\mathbf{M}_{\nu}$. Hence, this makes clear the advantage of working with the $\mathbf{Q}_{6}$ flavor symmetry. Let us go back to $\mathbf{M}_{\nu}$ matrix, the sub-block matrix $2-3$ provides a $45^{\circ}$ angle (to the flavor mixing matrix) that may be identified with the atmospheric one. Moreover, if the equality $m_{e \tau}=m_{e \mu}$ were true, the neutrino mass matrix, $\mathbf{M}_{\nu}^{\mathbf{0}}$, would possess the $\mu-\tau$ symmetry. In addition, the $\mathbf{M}_{\nu}^{\mathbf{0}}$ matrix would be diagonalized by means of the unitary transformation $\mathbf{U}_{\nu}^{0 \dagger} \mathbf{M}_{\nu}^{0} \mathbf{U}_{\nu}^{0}=\boldsymbol{\Delta}_{\nu}^{0}$, where

$$
\mathbf{U}_{\nu}^{0}=\left(\begin{array}{ccc}
\cos \theta_{\nu} & \sin \theta_{\nu} & 0 \\
-\frac{\sin \theta_{\nu}}{\sqrt{2}} & \frac{\cos \theta_{\nu}}{\sqrt{2}} & -\frac{1}{\sqrt{2}} \\
-\frac{\sin \theta_{\nu}}{\sqrt{2}} & \frac{\cos \theta_{\nu}}{\sqrt{2}} & \frac{1}{\sqrt{2}}
\end{array}\right) \quad \text { and } \quad \Delta_{\nu}^{0}=\operatorname{diag}\left(m_{\nu_{1}}^{0}, m_{\nu_{2}}^{0}, m_{\nu_{3}}^{0}\right)
$$

So, the matrix elements of $\mathbf{M}_{\nu}^{0}$ may be written in terms of neutrino mass eigenvalues and the $\theta_{\nu}$ angle as:

$$
\begin{array}{ll}
m_{e e}=\left(m_{\nu_{1}}^{0} \cos ^{2} \theta_{\nu}+m_{\nu_{2}}^{0} \sin ^{2} \theta_{\nu}\right), & m_{e \mu}=\frac{\sin 2 \theta_{\nu}\left(m_{\nu_{2}}^{0}-m_{\nu_{1}}^{0}\right)}{\sqrt{8}} \\
m_{\mu \mu}+m_{\mu \tau}=m_{\nu_{1}}^{0} \sin ^{2} \theta_{\nu}+m_{\nu_{2}}^{0} \cos ^{2} \theta_{\nu}, & m_{\mu \mu}-m_{\mu \tau}=m_{\nu_{3}}^{0}
\end{array}
$$

Strictly speaking, in the present model, $\mathbf{M}_{\nu}$ does not possess the $\mu \leftrightarrow \tau$ symmetry since $m_{e \mu} \neq m_{e \tau}$. This fact, actually, is crucial to get $\theta_{13} \neq 0^{\circ}$ and $\theta_{23} \neq 45^{\circ}$ in the PMNS matrix as we will see next. Now, the $\mathbf{M}_{\nu}$ mass matrix will be diagonalized in a perturbative way, as follows: applying $\mathbf{U}_{\nu}^{0}$ to $\mathbf{M}_{\nu}$, we have

$$
\mathbf{U}_{\nu}^{0 \dagger} \mathbf{M}_{\nu} \mathbf{U}_{\nu}^{0 *}=\boldsymbol{\Delta}_{\nu}^{0}+\left(\begin{array}{ccc}
0 & 0 & \frac{\cos \theta_{\nu}}{\sqrt{2}}\left(m_{e \tau}-m_{e \mu}\right) \\
\frac{\sin \theta_{\nu}}{\sqrt{2}}\left(m_{e \tau}-m_{e \mu}\right) \\
\frac{\cos \theta_{\nu}}{\sqrt{2}}\left(m_{e \tau}-m_{e \mu}\right) & \frac{\sin \theta_{\nu}}{\sqrt{2}}\left(m_{e \tau}-m_{e \mu}\right) & 0
\end{array}\right)
$$


The right side of the above expression contains the difference $m_{e \tau}-m_{e \mu}$ that breaks the $\mu-\tau$ symmetry. Then, this mass matrix will be considered as a perturbation to the $\mathbf{M}_{\nu}^{0}$ matrix. As a consequence, the $m_{\nu_{i}}^{0}$ physical neutrino masses will get a correction as well as the $\mathbf{U}_{\nu}^{0}$ neutrino mixing. Here, we define the dimensionless perturbation parameter as

$$
\epsilon \equiv \frac{\left(m_{e \tau}-m_{e \mu}\right)}{m_{e \mu}}
$$

where $|\epsilon| \ll 1$ as hypothesis. To be more precise, we will consider a soft breaking of the $\mu-\tau$ symmetry such that $|\epsilon| \ll 0.3$. Then, quadratic contributions on $\epsilon$ will be neglected along the analysis. In this way, the second matrix of right side of Eq. (15) is given as

$$
\mathbf{M}_{\nu}^{\epsilon}=\left(\begin{array}{ccc}
0 & 0 & \frac{\cos \theta_{\nu}}{\sqrt{2}} m_{e \mu} \epsilon \\
0 & 0 & \frac{\sin \theta_{\nu}}{\sqrt{2}} m_{e \mu} \epsilon \\
\frac{\cos \theta_{\nu}}{\sqrt{2}} m_{e \mu} \epsilon & \frac{\sin \theta_{\nu}}{\sqrt{2}} m_{e \mu} \epsilon & 0
\end{array}\right)
$$

In general, the $\mathbf{M}_{\nu}$ mass matrix may be diagonalized by means of a unitary transformation $\mathbf{U}_{\nu}^{\dagger} \mathbf{M}_{\nu} \mathbf{U}_{\nu}^{*}=\boldsymbol{\Delta}_{\nu}$, where $\boldsymbol{\Delta}_{\nu}=\operatorname{diag}\left(m_{\nu_{1}}, m_{\nu_{2}}, m_{\nu_{3}}\right), \mathbf{U}_{\nu} \approx \mathbf{U}_{\nu}^{0} \mathbf{U}_{\nu}^{\epsilon}$ in this latter the $\mathbf{U}_{\nu}^{0}$ matrix diagonalizes to $\mathbf{M}_{\nu}^{0}$, while $\mathbf{U}_{\nu}^{\epsilon}$ makes the same for the resultant matrix that depends on $\epsilon$. Here, the $m_{\nu_{i}}^{0}$ active neutrino masses are complex due to the presence of Majorana phases, and the $\theta_{\nu}$ angle is a free parameter. The explicit form of the unitary matrix $\mathbf{U}_{\nu}^{\epsilon}$ is the following

$$
\mathbf{U}_{\nu}^{\epsilon} \approx\left(\begin{array}{ccc}
N_{1}^{-1} & 0 & \frac{k_{1} r_{1} \epsilon}{N_{3}} \\
0 & N_{2}^{-1} & \frac{k_{2} r_{2} \epsilon}{N_{3}} \\
-\frac{k_{1} r_{1} \epsilon}{N_{1}} & -\frac{k_{2} r_{2} \epsilon}{N_{2}} & N_{3}^{-1}
\end{array}\right)
$$

where

$$
r_{1,2} \equiv\left(\frac{m_{\nu_{2}}^{0}-m_{\nu_{1}}^{0}}{m_{\nu_{3}}^{0}-m_{\nu_{1,2}}^{0}}\right), \quad k_{1} \equiv \frac{\sin 2 \theta_{\nu} \cos \theta_{\nu}}{4} \quad \text { and } \quad k_{2} \equiv \frac{\sin 2 \theta_{\nu} \sin \theta_{\nu}}{4}
$$

The normalization factors in the $\mathbf{U}_{\nu}^{\epsilon}$ are

$$
N_{1}=\sqrt{1+\left|\epsilon k_{1} r_{1}\right|^{2}}, \quad N_{2}=\sqrt{1+\left|\epsilon k_{2} r_{2}\right|^{2}}, \quad N_{3}=\sqrt{1+|\epsilon|^{2}\left(\left|k_{1} r_{1}\right|^{2}+\left|k_{2} r_{2}\right|^{2}\right)} .
$$

Finally, the lepton flavor mixing matrix is given as $\mathbf{V} \approx \mathbf{U}_{\ell}^{\dagger} \mathbf{U}_{\nu}^{0} \mathbf{U}_{\nu}^{\epsilon}$, where $\mathbf{U}_{\ell}$ for this theoretical framework is equal to the unity matrix, while $\mathbf{U}_{\nu}^{0}$ and $\mathbf{U}_{\nu}^{\delta}$ matrices are given in Eqs. 13 and 18 , respectively. Thus, we obtain

$$
\mathbf{V} \approx\left(\begin{array}{ccc}
\frac{\cos \theta_{\nu}}{N_{1}} & \frac{\sin \theta_{\nu}}{N_{2}} & \frac{\epsilon \cos ^{2} \theta_{\nu} \sin 2 \theta_{\nu} r_{1}\left(1+\frac{r_{2}}{r_{1}} \tan ^{2} \theta_{\nu}\right)}{4 N_{3}} \\
-\frac{\sin \theta_{\nu}\left(1-\epsilon\left(\cos ^{2} \theta_{\nu} / 2\right) r_{1}\right)}{\sqrt{2} N_{1}} & \frac{\cos \theta_{\nu}\left(1+\epsilon\left(\sin ^{2} \theta_{\nu} / 2\right) r_{2}\right)}{\sqrt{2} N_{2}} & -\frac{1-\epsilon\left(\sin ^{2} 2 \theta_{\nu} / 8\right) r_{1} r_{2}}{\sqrt{2} N_{3}} \\
-\frac{\sin \theta_{\nu}\left(1+\epsilon\left(\cos ^{2} \theta_{\nu} / 2\right) r_{1}\right)}{\sqrt{2} N_{1}} & \frac{\cos \theta_{\nu}\left(1-\epsilon\left(\sin ^{2} \theta_{\nu} / 2\right) r_{2}\right)}{\sqrt{2} N_{2}} & \frac{1+\epsilon\left(\sin ^{2} 2 \theta_{\nu} / 8\right) r_{1} r_{2}}{\sqrt{2} N_{3}}
\end{array}\right) .
$$

In order to obtain the theoretical expressions for the flavor mixing angles, we compare the magnitude of the entries in the mixing matrix PMNS given in the above parametrization and the Standard parametrization [54]. Then, in this theoretical framework the reactor, solar and atmospheric mixing angles have the form:

$$
\sin ^{2} \theta_{13}=\left|\mathbf{V}_{13}\right|^{2}=\frac{|\epsilon|^{2} \sin ^{2} 2 \theta_{\nu} \cos ^{4} \theta_{\nu}\left|r_{1}\right|^{2}\left|1+\frac{r_{2}}{r_{1}} \tan ^{2} \theta_{\nu}\right|^{2}}{16 N_{3}^{2}},
$$




$$
\begin{gathered}
\sin ^{2} \theta_{23}=\frac{\left|\mathbf{V}_{23}\right|^{2}}{1-\left|\mathbf{V}_{13}\right|^{2}}=\frac{1}{2 N_{3}^{2}} \frac{\left|1-\frac{\sin ^{2} 2 \theta_{\nu}}{8} \epsilon r_{1} r_{2}\right|^{2}}{1-\sin ^{2} \theta_{13}} \\
\sin ^{2} \theta_{12}=\frac{\left|\mathbf{V}_{12}\right|^{2}}{1-\left|\mathbf{V}_{13}\right|^{2}}=\frac{1}{N_{2}^{2}} \frac{\sin ^{2} \theta_{\nu}}{1-\sin ^{2} \theta_{13}}
\end{gathered}
$$

In the limit when $\epsilon$ goes to zero, one recovers the well known results of exact $\mu-\tau$ symmetry: $\theta_{13}=0^{\circ}$ and $\theta_{23}=45^{\circ}$. On the other hand, in order to figure out the mixing angles values that our model predicts, an analytic study will be done. To do this, we should keep in mind that $|\epsilon| \ll 1$, to be more precisely $|\epsilon| \leq 0.3$. With this in mind, the normalization factors should be of order of 1 . Then the expression for the solar mixing angle to leading order is

$$
\sin ^{2} \theta_{12} \approx \sin ^{2} \theta_{\nu} \quad \Rightarrow \quad \theta_{12} \approx \theta_{\nu}
$$

Thus, along the analytical study, we will consider that $\sin \theta_{\nu} \approx 1 / \sqrt{3}$ which is a good approximation to the solar angle [55]58]. The other two mixing angles take the form

$$
\sin ^{2} \theta_{23} \approx \frac{1}{2}\left|1-\frac{\epsilon}{8} \sin ^{2} 2 \theta_{12} r_{1} r_{2}\right|^{2} \quad \text { and } \quad \sin ^{2} \theta_{13} \approx \frac{|\epsilon|^{2}}{16} \sin ^{2} 2 \theta_{12} \cos ^{4} \theta_{12}\left|r_{1}\right|^{2}\left|1+\frac{r_{2}}{r_{1}} \tan ^{2} \theta_{12}\right|^{2} .
$$

The explicit form of the $r_{1,2}$ parameters are given in Eq. (19). Notice that the reactor angle as well as the atmospheric one depend strongly on the complex neutrino masses, so that the Majorana phases may be relevant to enhance or suppress those.

In order to show this last fact, the diagonal matrix, given in Eq. 13 , can be written as $\boldsymbol{\Delta}_{\nu}^{0}=$ $\operatorname{diag}\left(\left|m_{\nu_{1}}^{0}\right| e^{i \alpha_{1}},\left|m_{\nu_{2}}^{0}\right| e^{i \alpha_{2}},\left|m_{\nu_{3}}^{0}\right| e^{i \alpha_{3}}\right)$ where $\alpha_{i}$ is the respective Majorana phase for each neutrino mass. In the following study, we will consider the case of CP parities ( 0 or $\pi$ ) in the Majorana phases and some combinations among them. Then, we end up having $\boldsymbol{\Delta}_{\nu}^{0}=\operatorname{diag}\left( \pm\left|m_{\nu_{1}}^{0}\right|, \pm\left|m_{\nu_{2}}^{0}\right|, \pm\left|m_{\nu_{3}}^{0}\right|\right)$. As we will see, there are several cases where these CP parities play an important role to constrain the $\epsilon$ parameter and the lightest neutrino mass that accommodate the reactor and atmospheric angles. For the moment, none of the Majorana phases will be factorized so this analysis is equivalent up to one phase to the standard parametrization where two relative Majorana phases are taking into account.

In the last part of the work, as a particular prediction of this model, we will calculate the effective neutrino mass that comes from the neutrinoless double beta decay, the neutrino mass scale and the sum of the neutrino masses. These observables will be calculated in the framework CP parities and two relative Majorana phases; in the latter case, the analytical study is out of the scope of this work, but naive plots will be presented in order to compare them with the former case. For convenience, we will work with the parametrization $\boldsymbol{\Delta}_{\nu}^{0}=\operatorname{diag}\left(\left|m_{\nu_{1}}^{0}\right| e^{i \alpha},\left|m_{\nu_{2}}^{0}\right|,\left|m_{\nu_{3}}^{0}\right| e^{i \beta}\right) e^{i \alpha_{2}}$ where $\alpha \equiv \alpha_{1}-\alpha_{2}, \beta \equiv \alpha_{3}-\alpha_{2}$ and the $\alpha_{2}$ phase is irrelevant.

Let us start with the analytical study in the framework of CP parities.

1. Normal Hierarchy. From the definition of $\Delta m_{21}^{2}$ and $\Delta m_{31}^{2}$, one obtains the absolute masses $\left|m_{\nu_{3}}^{0}\right|=$ $\sqrt{\Delta m_{31}^{2}+m_{\nu_{1}}^{02}}$ and $\left|m_{\nu_{2}}^{0}\right|=\sqrt{\Delta m_{21}^{2}+m_{\nu_{1}}^{02}}$

$$
r_{1} \approx \frac{m_{\nu_{2}}^{0}}{m_{\nu_{3}}^{0}}\left(1-\frac{m_{\nu_{1}}^{0}}{m_{\nu_{2}}^{0}}\right), \quad \frac{r_{2}}{r_{1}} \approx 1+\frac{m_{\nu_{2}}^{0}}{m_{\nu_{3}}}, \quad r_{1} r_{2} \approx \frac{m_{\nu_{2}}^{0}}{m_{\nu_{3}}^{0}}\left(1+\frac{m_{\nu_{2}}^{0}}{m_{\nu_{3}}^{0}}\right)\left(1-\frac{m_{\nu_{1}}^{0}}{m_{\nu_{2}}^{0}}\right)^{2} .
$$


The above mass ratios will depend on the sign of $m_{\nu_{2}}^{0}$ and $m_{\nu_{3}}^{0}$. At the same time $m_{\nu_{2}}^{0} / m_{\nu_{3}}^{0} \approx \mathcal{O}\left(\sqrt{\Delta m_{12}^{2} / \Delta m_{31}^{2}}\right)$ up to some signs. Therefore, the reactor angle will be proportional to $\left(\Delta m_{12}^{2} / \Delta m_{31}^{2}\right)|\epsilon|^{2}$ which turns out being tiny. This statement holds to whatever signs are assumed in the neutrino masses, $m_{\nu_{2}}^{0}$ and $m_{\nu_{3}}^{0}$. Because of this, we may infer the normal case is ruled out for $|\epsilon| \leq 0.3$.

2. Inverted Hierarchy. Following a similar analysis to the normal case, we have $\left|m_{\nu_{2}}^{0}\right|=$ $\sqrt{\Delta m_{31}^{2}+\Delta m_{21}^{2}+\left|m_{\nu_{3}}^{0}\right|^{2}}$ and $\left|m_{\nu_{1}}^{0}\right|=\sqrt{\Delta m_{13}^{2}+\left|m_{\nu_{3}}^{0}\right|^{2}}$. For the mass ratios, we have

$$
\begin{aligned}
r_{1} & \approx-\left(\frac{m_{\nu_{2}}^{0}-m_{\nu_{1}}^{0}}{m_{\nu_{1}}^{0}}\right)\left(1+\frac{m_{\nu_{3}}^{0}}{m_{\nu_{1}}^{0}}\right), \quad \frac{r_{2}}{r_{1}} \approx \frac{m_{\nu_{1}}^{0}}{m_{\nu_{2}}^{0}}\left[1-\frac{m_{\nu_{3}}^{0}\left(m_{\nu_{2}}^{0}-m_{\nu_{1}}^{0}\right)}{m_{\nu_{2}}^{0} m_{\nu_{1}}^{0}}\right], \\
r_{1} r_{2} & \approx \frac{\left(m_{\nu_{2}}^{0}-m_{\nu_{1}}^{0}\right)^{2}}{m_{\nu_{1}}^{0} m_{\nu_{2}}^{0}}\left[1+\frac{m_{\nu_{3}}^{0}\left(m_{\nu_{2}}^{0}+m_{\nu_{1}}^{0}\right)}{m_{\nu_{2}}^{0} m_{\nu_{1}}^{0}}\right] .
\end{aligned}
$$

Notice that these mass ratios are sensitive at the sign of the neutrino masses $m_{\nu_{i}}^{0}$. Actually, there are four independent scenarios: Case A with $m_{\nu_{i}}^{0}>0$; Case $\mathbf{B}$ with $m_{\nu_{(1,2)}}^{0}>0$ and $m_{\nu_{3}}^{0}<0$; Case $\mathbf{C}$ with $m_{\nu_{(2,3)}}^{0}>0$ and $m_{\nu_{1}}^{0}<0$; Case $\mathbf{D}$ with $m_{\nu_{2}}^{0}>0$ and $m_{\nu_{(1,3)}}^{0}<0$. The former cases can be written as

$$
\begin{aligned}
r_{1} & \approx-\left(\frac{\left|m_{\nu_{2}}^{0}\right|-\left|m_{\nu_{1}}^{0}\right|}{\left|m_{\nu_{1}}^{0}\right|}\right)\left(1 \pm \frac{\left|m_{\nu_{3}}^{0}\right|}{\left|m_{\nu_{1}}^{0}\right|}\right), \quad \frac{r_{2}}{r_{1}} \approx \frac{\left|m_{\nu_{1}}^{0}\right|}{\left|m_{\nu_{2}}^{0}\right|}\left[1 \mp \frac{\left|m_{\nu_{3}}^{0}\right|\left(\left|m_{\nu_{2}}^{0}\right|-\left|m_{\nu_{1}}^{0}\right|\right)}{\left|m_{\nu_{2}}^{0}\right|\left|m_{\nu_{1}}^{0}\right|}\right] ; \\
r_{1} r_{2} & \approx \frac{\left(\left|m_{\nu_{2}}^{0}\right|-\left|m_{\nu_{1}}^{0}\right|\right)^{2}}{\left|m_{\nu_{1}}^{0}\right|\left|m_{\nu_{2}}^{0}\right|}\left[1 \pm \frac{\left|m_{\nu_{3}}^{0}\right|\left(\left|m_{\nu_{2}}^{0}\right|+\left|m_{\nu_{1}}^{0}\right|\right)}{\left|m_{\nu_{2}}^{0}\right|\left|m_{\nu_{1}}^{0}\right|}\right],
\end{aligned}
$$

where the upper (lower) sign corresponds to the Case A (Case B). For the Case $\mathbf{C}$ (Case D) the corresponding sign is the upper (lower), and this is given by

$$
\begin{aligned}
r_{1} & \approx\left(\frac{\left|m_{\nu_{2}}^{0}\right|+\left|m_{\nu_{1}}^{0}\right|}{\left|m_{\nu_{1}}^{0}\right|}\right)\left(1 \mp \frac{\left|m_{\nu_{3}}^{0}\right|}{\left|m_{\nu_{1}}^{0}\right|}\right), \quad \frac{r_{2}}{r_{1}} \approx-\frac{\left|m_{\nu_{1}}^{0}\right|}{\left|m_{\nu_{2}}^{0}\right|}\left[1 \pm \frac{\left|m_{\nu_{3}}^{0}\right|\left(\left|m_{\nu_{2}}^{0}\right|+\left|m_{\nu_{1}}^{0}\right|\right)}{\left|m_{\nu_{2}}^{0}\right|\left|m_{\nu_{1}}^{0}\right|}\right] ; \\
r_{1} r_{2} & \approx-\frac{\left(\left|m_{\nu_{2}}^{0}\right|+\left|m_{\nu_{1}}^{0}\right|\right)^{2}}{\left|m_{\nu_{1}}^{0}\right|\left|m_{\nu_{2}}^{0}\right|}\left[1 \mp \frac{\left|m_{\nu_{3}}^{0}\right|\left(\left|m_{\nu_{2}}^{0}\right|-\left|m_{\nu_{1}}^{0}\right|\right)}{\left|m_{\nu_{2}}^{0}\right|\left|m_{\nu_{1}}^{0}\right|}\right] .
\end{aligned}
$$

From the absolute neutrino mass expressions, we notice that $\left|m_{\nu_{2}}^{0}\right| \approx\left|m_{\nu_{1}}^{0}\right|\left(1+R_{1}\right)$, then

$$
\left|m_{\nu_{2}}^{0}\right|-\left|m_{\nu_{1}}^{0}\right| \approx\left|m_{\nu_{1}}^{0}\right| R_{1}, \quad\left|m_{\nu_{2}}^{0}\right|+\left|m_{\nu_{1}}^{0}\right| \approx 2\left|m_{\nu_{1}}^{0}\right|\left(1+\frac{R_{1}}{2}\right), \quad\left|m_{\nu_{2}}^{0}\right|\left|m_{\nu_{1}}^{0}\right| \approx\left|m_{\nu_{1}}^{0}\right|^{2}\left(1+R_{1}\right),
$$

where $R_{1} \equiv \Delta m_{21}^{2} / 2\left|m_{\nu_{1}}^{0}\right|^{2} \sim 10^{-2}$ which is valid whereas the lightest neutrino mass will be tiny.

Roughly speaking, for the Cases $\mathbf{A}$ and $\mathbf{B}$, the mixing angles are similar and these are given as

$$
\sin ^{2} \theta_{13} \approx \frac{|\epsilon|^{2}}{18} R_{1}^{2}\left(1-\frac{2}{3} R_{1}\right), \quad \sin ^{2} \theta_{23} \approx \frac{1}{2}\left|1-\frac{\epsilon}{9} R_{1}^{2}\right|^{2} .
$$

These relations allow us to discard the Case $\mathbf{A}$ and $\mathbf{B}$ since the reactor angle is proportional to $|\epsilon|^{2} R_{1}^{2}$ that turns out too small for $|\epsilon| \leq 0.3$. In the Case $\mathbf{C}$ and $\mathbf{D}$, one obtains

$$
\sin ^{2} \theta_{13} \approx \frac{2}{81}|\epsilon|^{2}\left(1+3 R_{1} \mp 6 \frac{\left|m_{\nu_{3}}^{0}\right|}{\left|m_{\nu_{1}}^{0}\right|}\right), \quad \sin ^{2} \theta_{23} \approx \frac{1}{2}\left|1+\frac{4}{9} \epsilon\left(1 \mp \frac{\left|m_{\nu_{3}}^{0}\right|}{\left|m_{\nu_{1}}^{0}\right|} R_{1}\right)\right|^{2} .
$$

From these formulas, in the strict inverted hierarchy, $\left|m_{\nu_{3}}^{0}\right|=0$ then $R_{1} \rightarrow \Delta m_{21}^{2} / 2 \Delta m_{13}^{2}$, we obtain: 
(a) If $|\epsilon|=0.3$,

$$
\sin ^{2} \theta_{13} \approx 0.0023, \quad \sin ^{2} \theta_{23}=\left\{\begin{array}{lll}
0.64, & \text { with } & \alpha_{\epsilon}=0 \\
0.37, & \text { with } & \alpha_{\epsilon}=\pi
\end{array}\right.
$$

(b) If $|\epsilon|=0.1$,

$$
\sin ^{2} \theta_{13} \approx 0.00025, \quad \sin ^{2} \theta_{23}=\left\{\begin{array}{lll}
0.54, & \text { with } & \alpha_{\epsilon}=0 \\
0.45, & \text { with } & \alpha_{\epsilon}=\pi
\end{array}\right.
$$

In here, we emphasize the importance of the $|\epsilon| e^{i \alpha_{\epsilon}}$ associated phase of the perturbation parameter since this might lead to a deviation above or below of $45^{\circ}$ the atmospheric angle. Then, from above cases the atmospheric angle is accommodated in the allowed region with $\alpha_{\epsilon}=0$. Therefore, this value for the phase will be chosen when the lightest neutrino mass is very small but different from zero .

Going back to Eq. (33), we observe that the Case $\mathbf{C}$ will be disfavored since the reactor angle is reduced by the term $6\left|m_{\nu_{3}}^{0}\right| /\left|m_{\nu_{1}}^{0}\right|$; this statement can be verified in a straightforward way, as it will be done also for the Case $\mathbf{D}$. Fixing the reactor angle to its central value we can figure out the allowed values for the perturbation parameter and the lightest neutrino mass. Then, if $\left|m_{\nu_{3}}^{0}\right|=0.001$, one requires that $|\epsilon| \approx 0.89$ to get $\sin ^{2} \theta_{13}=0.0229$. As result, we obtain $\sin ^{2} \theta_{23} \approx 0.97$ which is large in comparison to the allowed values. Now, if $\left|m_{\nu_{3}}^{0}\right|=0.1$, we need that $|\epsilon| \approx 0.38$ to get $\sin ^{2} \theta_{13}=0.0229$, in this way, $\sin ^{2} \theta_{23} \approx 0.68$ which is on the top of the allowed experimental region. Remarkably, in this brief analysis the value of the perturbation parameter and the lightest neutrino mass are on the limit of soft breaking of the $\mu-\tau$ symmetry and the degenerate region, respectively. The second plot of figure 2 shows how $\left|m_{\nu_{3}}^{0}\right|$ depends on $|\epsilon|$ and viceversa, which is consistent with the previous analysis. In addition, notice that the reactor angle prefers large values for $|\epsilon|$ whereas the atmospheric one is favored with small values.

In order to figure out the parameter space that fits the best values of the reactor and atmospheric angles, the exact formulas for these were taken and the observables as $\Delta m_{21}^{2}, \Delta m_{13}^{2}$ and $\theta_{\nu}$ were considered up to $3 \sigma$. With this in mind, in the following plots for the inverted and degenerate hierarchy, we demand that the reactor angle within $3 \sigma$ of C. L. of its experimental values (see the first plot in figure 1) to determine and constrain the atmospheric angle and the free parameters $|\epsilon|$ and $\left|m_{\nu_{3}}^{0}\right|$, respectively. The set of values for these parameters are shown in the next plots.

3. Degenerate Hierarchy. In this case, we have that $\left|m_{\nu_{1}}^{0}\right| \approx\left|m_{\nu_{2}}^{0}\right| \approx\left|m_{\nu_{3}}^{0}\right| \approx m_{0}$ with $m_{0} \gtrsim 0.1 \mathrm{eV}$. Then, the absolute neutrino masses are $\left|m_{\nu_{3}}^{0}\right|=\sqrt{\Delta m_{31}^{2}+m_{0}^{2}} \approx m_{0}\left(1+\Delta m_{31}^{2} / 2 m_{0}^{2}\right)$ and $\left|m_{\nu_{2}}^{0}\right|=\sqrt{\Delta m_{21}^{2}+m_{0}^{2}} \approx$ $m_{0}\left(1+\Delta m_{21}^{2} / 2 m_{0}^{2}\right)$. As in the inverted case, the extreme Majorana phases in the neutrino masses are relevant for the mixing angles, as we will see next. There are four independent cases for the signs which are shown below.

- Case A. If $m_{\nu_{i}}^{0}>0$, then we have that

$$
r_{1}=\frac{\left|m_{\nu_{2}}^{0}\right|-m_{0}}{\left|m_{\nu_{3}}^{0}\right|-m_{0}}, \quad r_{1} r_{2}=\frac{\left(\left|m_{\nu_{2}}^{0}\right|-m_{0}\right)^{2}}{\left(\left|m_{\nu_{3}}^{0}\right|-\left|m_{\nu_{2}}^{0}\right|\right)\left(\left|m_{\nu_{3}}^{0}\right|-m_{0}\right)}, \quad \frac{r_{2}}{r_{1}}=\frac{\left|m_{\nu_{3}}^{0}\right|-m_{0}}{\left|m_{\nu_{3}}^{0}\right|-\left|m_{\nu_{2}}^{0}\right|} .
$$

- Case B. If $m_{\nu_{(1,2)}}^{0}>0$ and $m_{\nu_{3}}^{0}<0$. As result of this, we get

$$
r_{1}=-\frac{\left|m_{\nu_{2}}^{0}\right|-m_{0}}{\left|m_{\nu_{3}}^{0}\right|+m_{0}}, \quad r_{1} r_{2}=\frac{\left(\left|m_{\nu_{2}}^{0}\right|-m_{0}\right)^{2}}{\left(\left|m_{\nu_{3}}^{0}\right|+\left|m_{\nu_{2}}^{0}\right|\right)\left(\left|m_{\nu_{3}}^{0}\right|+m_{0}\right)}, \quad \frac{r_{2}}{r_{1}}=\frac{\left|m_{\nu_{3}}^{0}\right|+m_{0}}{\left|m_{\nu_{3}}^{0}\right|+\left|m_{\nu_{2}}^{0}\right|} .
$$



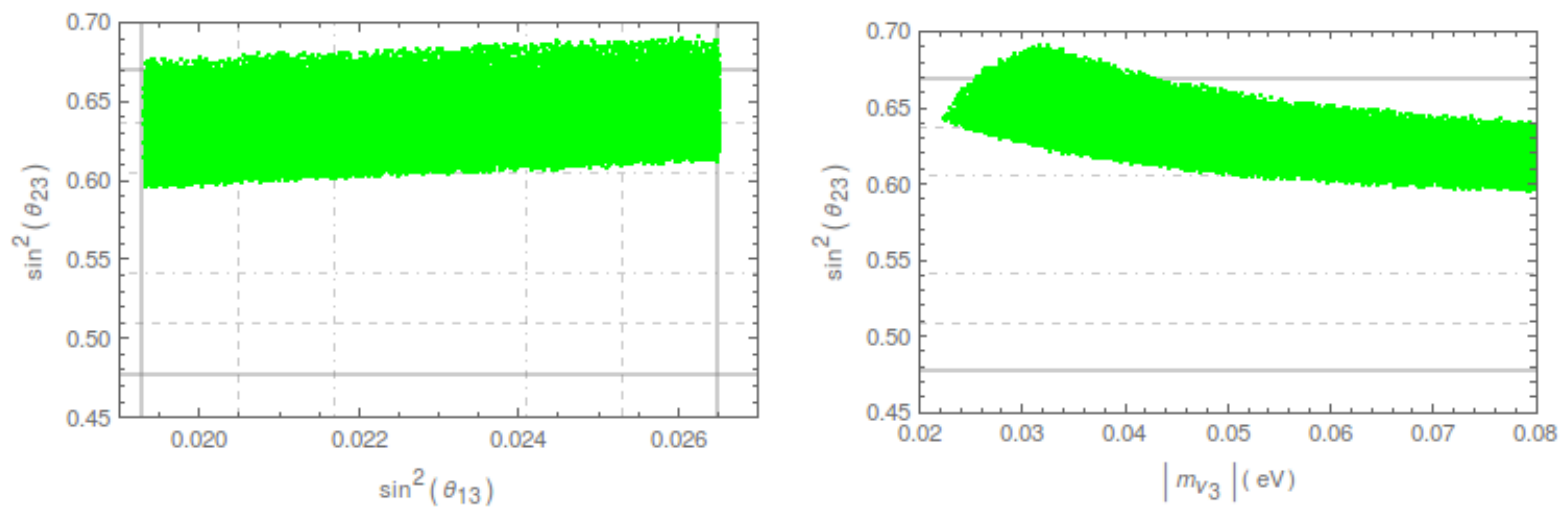

FIG. 1: Case D: Allowed region for $\sin ^{2} \theta_{13}$ and $\sin ^{2} \theta_{23}$, respectively. The dotdashed, dashed and thick lines stand for $1 \sigma$, $2 \sigma$ and $3 \sigma$, respectively
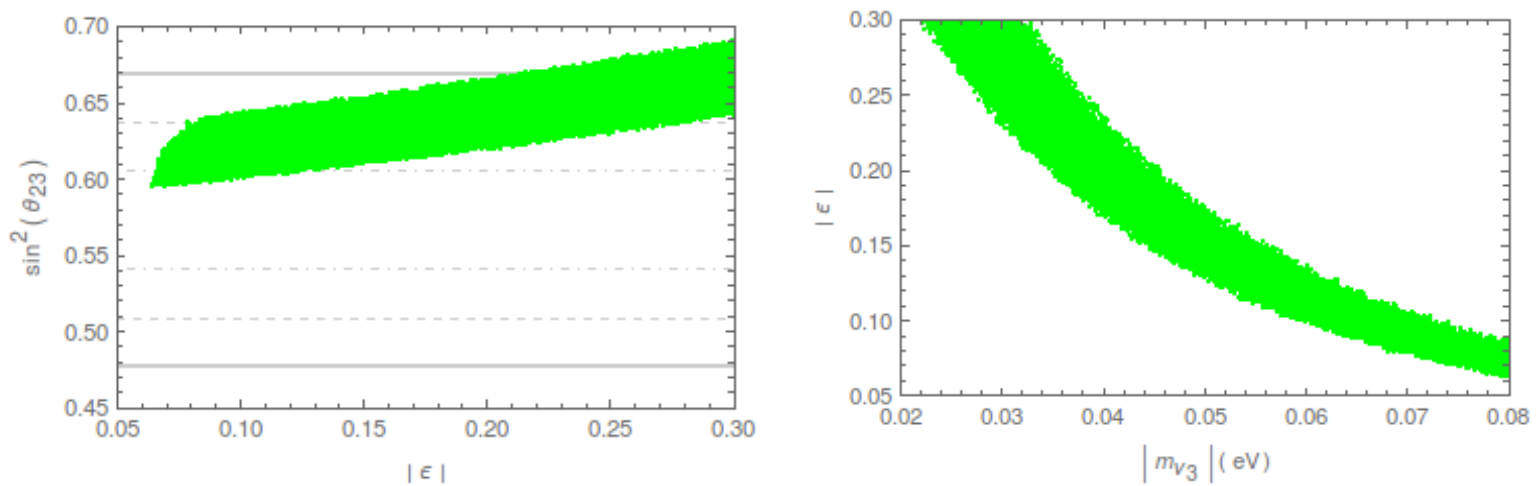

FIG. 2: Case D: Allowed region for $\sin ^{2} \theta_{23}$ versus $|\epsilon|$ and $|\epsilon|$ versus $\left|m_{\nu_{3}}^{0}\right|$. The dotdashed, dashed and thick lines stand for $1 \sigma, 2 \sigma$ and $3 \sigma$, respectively

- Case C. If $m_{\nu_{(2,3)}}^{0}>0$ and $m_{\nu_{1}}^{0}=-m_{0}$, therefore one obtains

$$
r_{1}=\frac{\left|m_{\nu_{2}}^{0}\right|+m_{0}}{\left|m_{\nu_{3}}^{0}\right|+m_{0}}, \quad r_{1} r_{2}=\frac{\left(\left|m_{\nu_{2}}^{0}\right|+m_{0}\right)^{2}}{\left(\left|m_{\nu_{3}}^{0}\right|-\left|m_{\nu_{2}}^{0}\right|\right)\left(\left|m_{\nu_{3}}^{0}\right|+m_{0}\right)}, \quad \frac{r_{2}}{r_{1}}=\frac{\left|m_{\nu_{3}}^{0}\right|+m_{0}}{\left|m_{\nu_{3}}^{0}\right|-\left|m_{\nu_{2}}^{0}\right|} .
$$

- Case D. If $m_{\nu_{2}}^{0}>0$ and $m_{\nu_{(1,3)}}^{0}<0$, then we have

$$
r_{1}=-\frac{\left|m_{\nu_{2}}^{0}\right|+m_{0}}{\left|m_{\nu_{3}}^{0}\right|-m_{0}}, \quad r_{1} r_{2}=\frac{\left(\left|m_{\nu_{2}}^{0}\right|+m_{0}\right)^{2}}{\left(\left|m_{\nu_{3}}^{0}\right|+\left|m_{\nu_{2}}^{0}\right|\right)\left(\left|m_{\nu_{3}}^{0}\right|-m_{0}\right)}, \quad \frac{r_{2}}{r_{1}}=\frac{\left|m_{\nu_{3}}^{0}\right|-m_{0}}{\left|m_{\nu_{3}}^{0}\right|+\left|m_{\nu_{2}}^{0}\right|} .
$$

Now, observe that

$$
\begin{array}{ll}
\left|m_{\nu_{2}}^{0}\right|-m_{0} \approx 2 m_{0} R_{2}, & \left|m_{\nu_{2}}^{0}\right|+m_{0} \approx 2 m_{0}\left(1+R_{2}\right), \\
\left|m_{\nu_{3}}^{0}\right|-m_{0} \approx 2 m_{0} R_{3}, & \left|m_{\nu_{3}}^{0}\right|+m_{0} \approx 2 m_{0}\left(1+R_{3}\right), \\
\left|m_{\nu_{3}}^{0}\right|-\left|m_{\nu_{2}}^{0}\right| \approx 2 m_{0}\left(R_{3}-R_{2}\right), & \left|m_{\nu_{3}}^{0}\right|+\left|m_{\nu_{2}}^{0}\right| \approx 2 m_{0}\left[1+R_{2}+R_{3}\right] .
\end{array}
$$

Here, $R_{2}=\Delta m_{21}^{2} / 4 m_{0}^{2}$ and $R_{3}=\Delta m_{31}^{2} / 4 m_{0}^{2}$, then, $R_{2} \ll R_{3}$. From these expressions, in the Case $\mathbf{A}$ and $\mathbf{B}$, we obtain respectively

$$
\begin{array}{ll}
\sin ^{2} \theta_{13} \approx \frac{|\epsilon|^{2}}{18}\left(\frac{R_{2}}{R_{3}}\right)^{2}\left(1+\frac{2}{3} \frac{R_{2}}{R_{3}}\right), & \sin ^{2} \theta_{23} \approx \frac{1}{2}\left|1-\frac{\epsilon}{9} \frac{R_{2}}{R_{3}}\right|^{2} \\
\sin ^{2} \theta_{13} \approx \frac{|\epsilon|^{2}}{18} R_{2}^{2}\left[1+\frac{2}{3}\left(R_{2}+2 R_{3}\right)\right], & \sin ^{2} \theta_{23} \approx \frac{1}{2}\left|1-\frac{\epsilon}{9} R_{2}^{2}\right|^{2} .
\end{array}
$$


Both cases are discarded since the reactor angle is proportional to the small quantities $\left(R_{2} / R_{3}\right)^{2} \approx \mathcal{O}\left(10^{-3}\right)$ and $\left(R_{2}\right)^{2} \approx \mathcal{O}\left(10^{-5}\right)$, respectively. Here, we have taken the central values for the inverted hierarchy and $m_{0} \approx 0.1 \mathrm{eV}$, in addition $1 / R_{3} \approx 16$ and $|\epsilon| \leq 0.3$. In fact, the values of $R_{2}$ and $R_{3}$ might be tiny if $m_{0}$ is large.

In the Cases $\mathbf{C}$ and $\mathbf{D}$, we have respectively

$$
\begin{aligned}
& \sin ^{2} \theta_{13} \approx \frac{|\epsilon|^{2}}{18}\left(1+2 R_{2}-2 R_{3}\right)\left(1+\frac{1}{3 R_{3}}\right)^{2}, \quad \sin ^{2} \theta_{23} \approx \frac{1}{2}\left|1-\frac{\epsilon}{9}\left(\frac{1+R_{2}-R_{3}}{R_{3}}\right)\right|^{2}, \\
& \sin ^{2} \theta_{13} \approx \frac{2}{81}|\epsilon|^{2}\left(1+R_{3}\right)\left(\frac{1+R_{2}}{R_{3}}\right)^{2}, \quad \sin ^{2} \theta_{23} \approx \frac{1}{2}\left|1-\frac{\epsilon}{9}\left(\frac{1+R_{2}-R_{3}}{R_{3}}\right)\right|^{2} .
\end{aligned}
$$

As can be noticed, in the Case $\mathbf{D}$, the reactor angle is proportional to $\left(1 / R_{3}\right)^{2}$, which is a large quantity so that one requires that $|\epsilon|$ should be $10^{-2}$ to obtain the region where the atmospheric angle lies on. This favors the atmospheric angle since small values of $|\epsilon|$ are needed to not deviate too much from $45^{\circ}$. On the contrary, in the Case $\mathbf{C}$ the atmospheric angle is deviated considerably since the $|\epsilon|$ value is large, in comparison to the above case, thus it is not necessary to suppress too much the $\left(1+1 / 3 R_{3}\right)^{2}$ factor in the reactor angle.

In a similar way to the inverted hierarchy, fixing the reactor angle to its central value we obtain the following for the Case C:

(a) If $m_{0}=0.1 \mathrm{eV}$

$$
\sin ^{2} \theta_{13}=0.0229, \quad \sin ^{2} \theta_{23} \approx\left\{\begin{array}{l}
0.33, \quad \text { with } \quad \alpha_{\epsilon}=0, \quad \text { and } \quad|\epsilon| \approx 0.1 \\
0.698, \quad \text { with } \quad \alpha_{\epsilon}=\pi, \quad \text { and } \quad|\epsilon| \approx 0.1
\end{array}\right.
$$

(b) If $m_{0}=0.25 \mathrm{eV}$

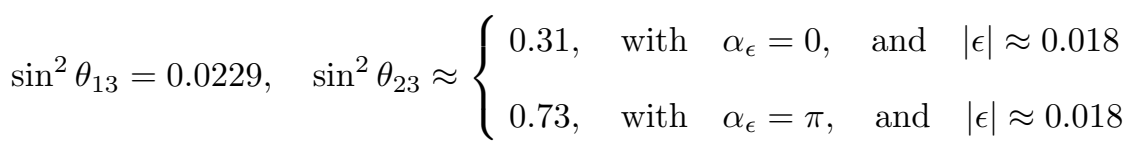

In the Case D, we obtain

(a) If $m_{0}=0.1 \mathrm{eV}$

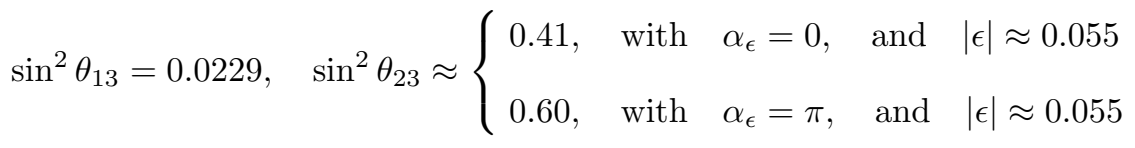

(b) If $m_{0}=0.25 \mathrm{eV}$

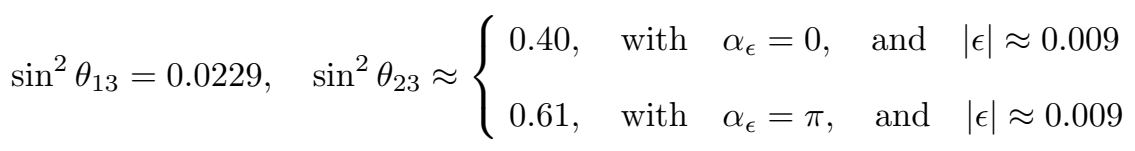

From the above results, we point out the importance of the $\alpha_{\epsilon}$ phase of the parameter $\epsilon$; this has to be $\pi$ in order to reach the allowed region for the atmospheric angle. Analogously to the inverted ordering, on the other hand, those results show the dependence between $m_{0}$ and $|\epsilon|$, the full allowed region is shown in the second plot of figure 4 .

To get a panoramic view of the parameter space, the exact formulas for the reactor and atmospheric angles were taken for the Case $\mathbf{D}$ and the observables as $\Delta m_{21}^{2}, \Delta m_{13}^{2}$ and $\theta_{\nu}=\theta_{12}$ were considered up to $3 \sigma$. For the free parameters $|\epsilon|$ and $m_{0}$, the set of values is shown in the following plots. As we can see, the reactor and atmospheric angles are accommodated in good agreement with their experimental values. 

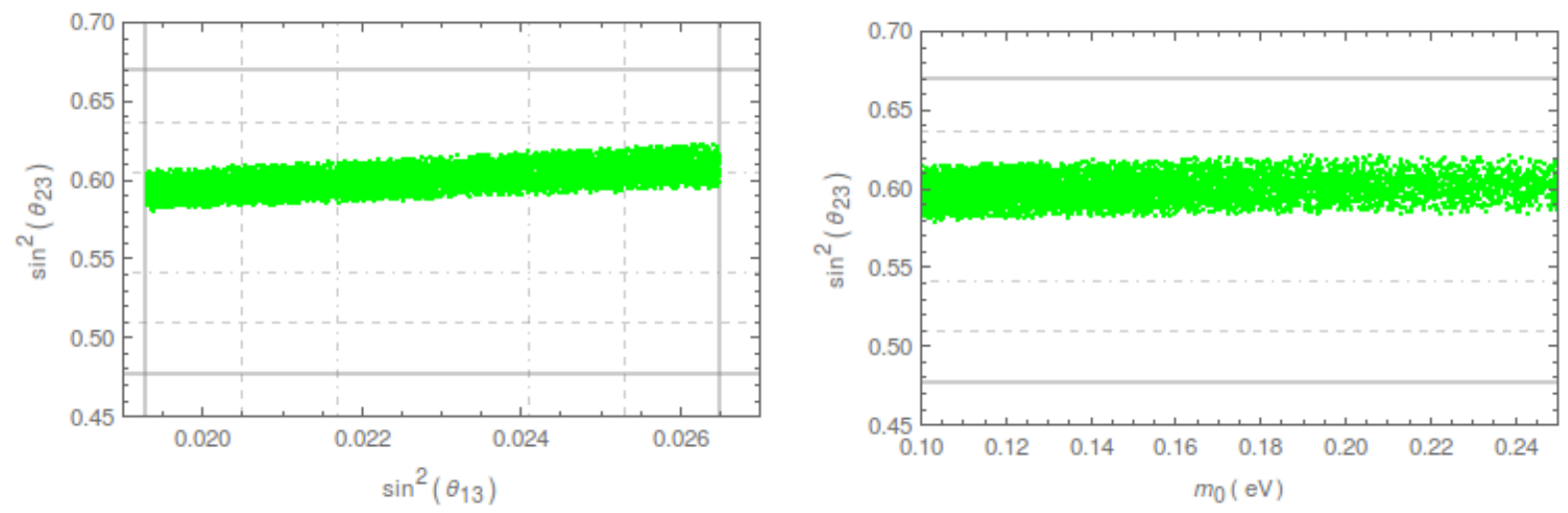

FIG. 3: Case D: Allowed region for $\sin ^{2} \theta_{13}$ and $\sin ^{2} \theta_{23}$, respectively. The dotdashed, dashed and thick lines stand for $1 \sigma$, $2 \sigma$ and $3 \sigma$, respectively for each case.
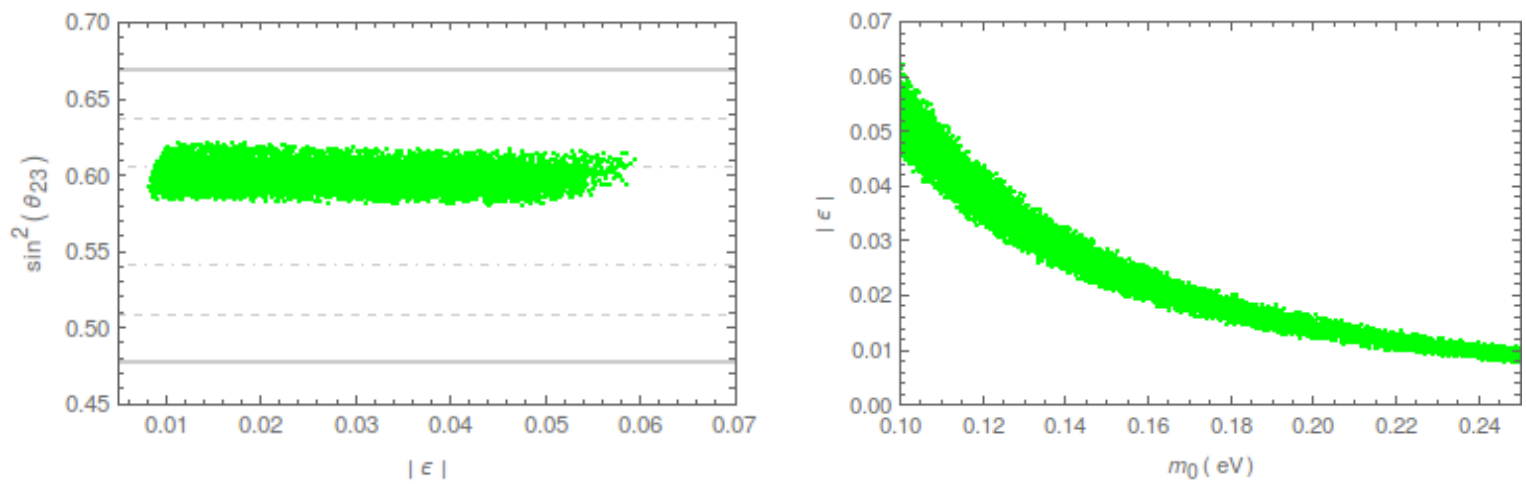

FIG. 4: Case D: Allowed region for $\sin ^{2} \theta_{23}$ versus $|\epsilon|$ and $|\epsilon|$ versus $m_{0}$. The dotdashed, dashed and thick lines stand for $1 \sigma, 2 \sigma$ and $3 \sigma$, respectively

\section{PREDICTION ON THE ABSOLUTE NEUTRINO MASS SCALE}

From the neutrino oscillation experiments, we get information on the mass square differences, but these experiments cannot say anything about the absolute neutrino mass scale. However, there are three processes that can address directly the determination of this important parameter: $i$ ) analysis of CMB temperature fluctuations [59], ii) the single $\beta$ decay [60] and iii) neutrinoless double beta decay $(0 \nu \beta \beta)$ [61. The first one is purely observational and the neutrino masses are determined with kinematical methods. The quantity probed in this approach is the sum of active neutrino masses, which we can denote as:

$$
m_{\mathrm{cosm}}=\sum_{i=1}^{3} m_{\nu_{i}}
$$

An upper bound of $m_{\text {cosm }}$ has been estimated by the Planck collaboration, and its value is $m_{\operatorname{cosm}}=\sum_{i}\left|m_{\nu_{i}}\right|=$ $0.23 \mathrm{eV}$ [62. However, this method is called model dependent because it has a strong dependence on the cosmological and astrophysical assumptions [59].

On the other hand, the second and third processes for the determination of absolute neutrino mass scale, which we will study in this work, are based on the searches conducted in ground laboratories [60, 61]. In the $\beta$ decay processes, 
for example in the tritium decay, the effective electron mass is defined as [1, 63, 67]:

$$
m_{\beta}^{2}=\sum_{i}^{3} m_{\nu_{i}}^{2}\left|V_{e i}\right|^{2},
$$

where $V_{e i}$ are the entries of lepton mixing matrix and correspond to the its first row. For a quasidegenerate neutrino mass spectrum $m_{\nu_{1}} \simeq m_{\nu_{2}} \simeq m_{\nu_{3}} \simeq m_{\nu_{e}}$, and for both possible hierarchies in the spectrum, the $m_{\beta}$ coincides approximately with the values of the three neutrino masses. Thus, Eq. 48 takes the form: $m_{\beta}^{2} \simeq m_{\nu_{e}}^{2} \sum_{i}^{3}\left|V_{e i}\right|^{2}=m_{\nu_{e}}^{2}$. The Mainz [68], Troitsk [69] and KATRIN [70] experiments can give us information on the absolute values of neutrino masses in the quasidegenerate region. From the first two experiments we have obtained an upper limit to the electron anti-neutrino mass of $2.3 \mathrm{eV}$. The KATRIN experiment was designed to improve this limit by one order of magnitude down, such that either it discovers its mass, or sets an upper limit of $0.2 \mathrm{eV} 70$ 72].

A direct measurement of the absolute neutrino mass scale through $\beta$ decay experiments, will not give us any information on the Dirac or Majorana character of neutrinos. The above issue can be solved by means of the neutrinoless double beta decay experiments $(0 \nu \beta \beta)$. This is because the $0 \nu \beta \beta$ decay is only possible if neutrinos are Majorana particles. Also, with this decay process we can probe the absolute neutrino mass scale by measuring of the effective Majorana mass of the electron neutrino, which is defined as:

$$
\left|m_{e e}\right|=\left|\sum_{i=1}^{3} m_{\nu_{i}} V_{e i}^{2}\right| .
$$

The lowest upper bound on $\left|m_{e e}\right|$ is provided by GERDA phase-I data [73], and this is $0.22 \mathrm{eV}$. That value will be substantially reduced by GERDA phase-II data [74].

Now, for our theoretical framework and in the case of CP parities, the Case $\mathbf{D}$ is the most favorable to accommodate the reactor and atmospheric angles for the inverted and degenerate hierarchy. Therefore, the above observables will be studied in this case. We have to keep in mind that the parameter that breaks the $\mu-\tau$ symmetry is small in both hierarchies so that the normalization factors are $N_{i} \approx \mathcal{O}(1)$. Due to CP parities the $\left|m_{e e}\right|$ effective neutrino mass becomes a real quantity, additionally there will be a cancellation among the involved terms; as a consequence, we expect small values in comparison to the GERDA phase-I data.

In the scatter plots that will be shown later, for certain quantities the green and red colors stand for the CP parities and the CP non trivial values for the Majorana phases, respectively. In order to get these plots, the previous method and the same conditions in determining the atmospheric angle and constraining the $|\epsilon|$ parameter and the lightest neutrino mass were used. This is, the exact formulas for the respective quantities are taken into account, additionally the observables as $\Delta m_{21}^{2}, \Delta m_{13}^{2}$ and $\theta_{12}$ were considered up to $3 \sigma$ of C.L. At the same time, the reactor angle was fixed to be consistent with the experimental value up to $3 \sigma$ of C. L. In this manner, we have calculated naively the following quantities in the presence of $\mathrm{CP}$ parities and $\mathrm{CP}$ non trivial values for the Majorana phases.

1. Inverted Hierarchy. The effective neutrino mass $\left|m_{e e}\right|$ is given by

$$
\left|m_{e e}\right| \approx \frac{1}{3}\left|m_{\nu_{1}}^{0}\right||1+3| \frac{m_{\nu_{3}}^{0}}{m_{\nu_{1}}^{0}}\left|\sin ^{2} \theta_{13}-R_{1}\right| .
$$

where the Eq. 21] has been used, $R_{1}=\Delta m_{21}^{2} / 2\left|m_{\nu_{1}}^{0}\right|^{2}$ and $\sin \theta_{\nu} \approx 1 / \sqrt{3}$. In the limit case of strict inverted ordering, $\left|m_{\nu_{3}}^{0}\right|=0$, one would have a defined value so we will expect that

$$
\left|m_{e e}\right|>\frac{\sqrt{\Delta m_{13}^{2}}}{3}\left[1-\frac{1}{2} \frac{\Delta m_{21}^{2}}{\Delta m_{13}^{2}}\right] \approx 0.016 \mathrm{eV} .
$$


Since the lightest neutrino mass is not allowed to be zero as can be seen in Fig. 1. According of the allowed values for the lightest neutrino mass and the $\epsilon$ parameter, the complete region of values for $\left|m_{e e}\right|$ is displayed in Fig. 5. In these plots, we have considered that $\alpha_{\epsilon}=0$ as was shown in the analytic study. In the case of
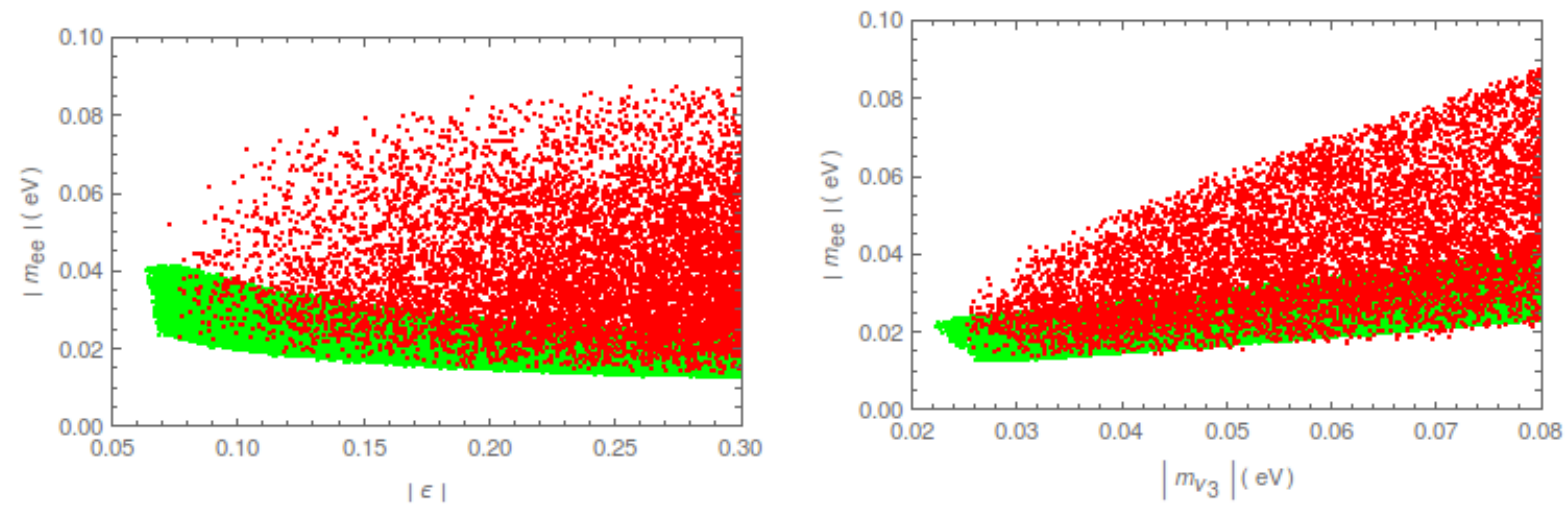

FIG. 5: Allowed region for $\left|m_{e e}\right|$ versus $\left|m_{\nu_{3}}^{0}\right|$ and $|\epsilon|$, respectively.

$\mathrm{CP}$ parities, the predicted region of values are below the lowest upper bound 73 . However, in the presence of $\mathrm{CP}$ non trivial values for the relative Majorana phases, $\alpha$ and $\beta$, the allowed region may be increased up to the region where GERDA phase-II data will be sensitive.

The neutrino mass scale in beta decays and the sum of neutrino masses are given respectively as

$$
m_{\nu_{e}} \approx\left|m_{\nu_{1}}^{0}\right|\left[1+\frac{1}{3} R_{1}+\frac{1}{2}\left|\frac{m_{\nu_{3}}^{0}}{m_{\nu_{1}}^{0}}\right|^{2} \sin ^{2} \theta_{13}\right], \quad \sum_{i}\left|m_{\nu_{i}}\right| \approx 2\left|m_{\nu_{1}}^{0}\right|\left[1+\frac{1}{2}\left(R_{1}+\left|\frac{m_{\nu_{3}}^{0}}{m_{\nu_{1}}^{0}}\right|\right)\right] .
$$

These observables are expected to be large than the limit values, $\left|m_{\nu_{3}}^{0}\right|=0$, this means

$$
m_{\nu_{e}}>\sqrt{\Delta m_{13}^{2}}\left[1+\frac{1}{6} \frac{\Delta m_{21}^{2}}{\Delta m_{13}^{2}}\right] \approx 0.049 \mathrm{eV}, \quad \sum_{i}\left|m_{\nu_{i}}\right|>2 \sqrt{\Delta m_{13}^{2}}\left[1+\frac{1}{2} \frac{\Delta m_{21}^{2}}{\Delta m_{13}^{2}}\right] \approx 0.098 \mathrm{eV} .
$$

The central values for the $\Delta m_{21}^{2}, \Delta m_{31}^{2}$ and the fixed reactor angle have been considered for this purpose. Notice the neutrino mass scale is below the value reported, $m_{\nu_{e}}=0.2 \mathrm{eV}$, as can be seen in Fig. 6, in the case of CP parities and $\mathrm{CP}$ non trivial values. However, the bound on the sum of the neutrino masses can be reached in both frameworks.
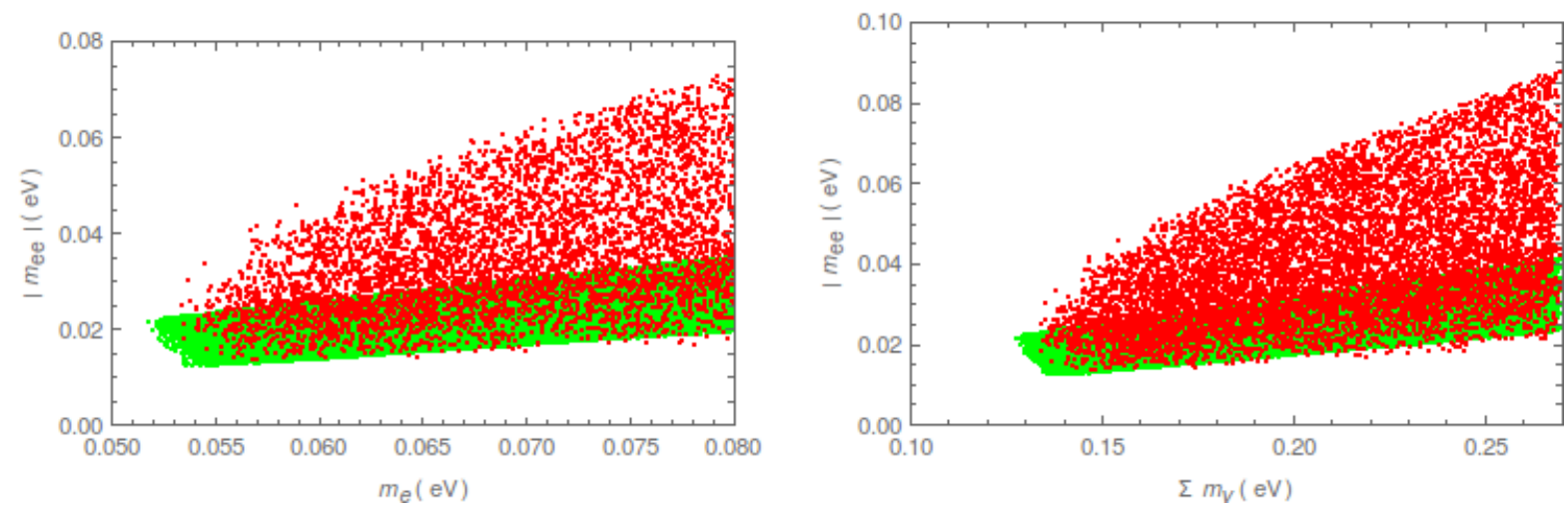

FIG. 6: Predicted region for $\left|m_{e e}\right|$ versus the neutrino mass scale $m_{\nu_{e}}$ and the sum of neutrino masses. 
To close this brief analysis, let us add two plots. The first one shows the allowed regions for the atmospheric angle against the relative Majorana phases $\alpha$ and $\beta$. According the analytical study, in the case of CP parities, the Case $\mathbf{D}$ is the most favorable to accommodate the mixing angles; this corresponds to $\alpha, \beta=\pi$. Along with this, in the second panel the effective neutrino mass is shown against the two relative Majorana phases. These plots are consistent with our analytical results obtained previously.
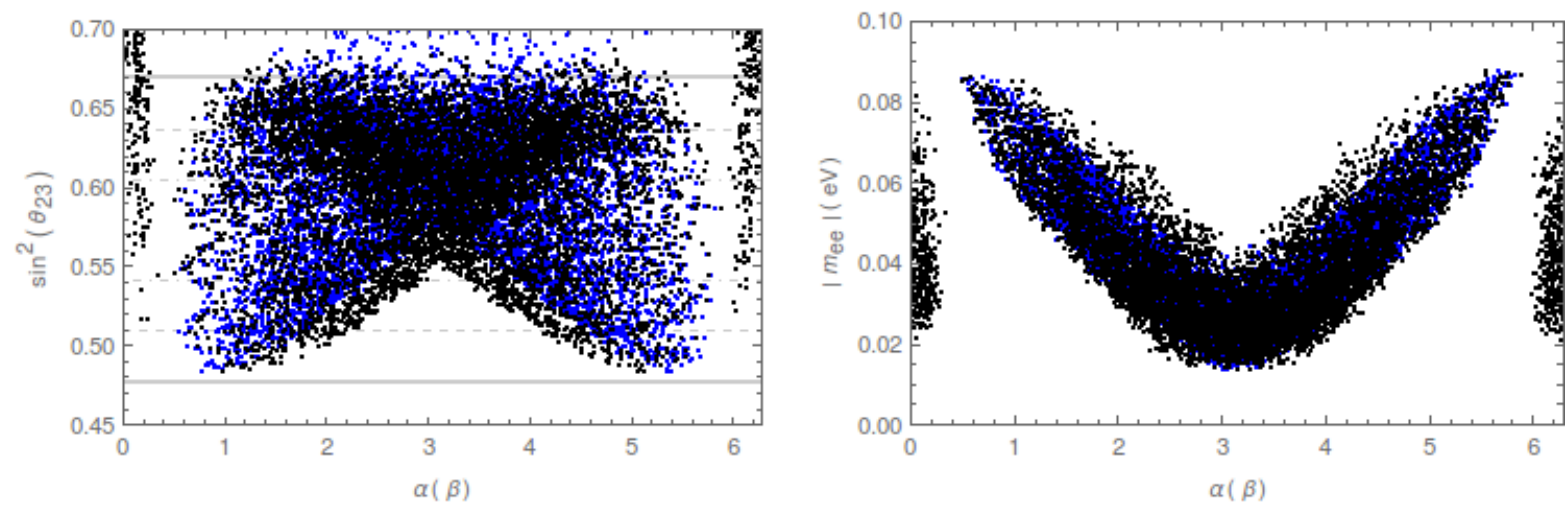

FIG. 7: Blue and black points stand for $\alpha$ and $\beta$ phases, respectively.

2. Degenerate Hierarchy. In this case, we obtain

$$
\left|m_{e e}\right| \approx \frac{m_{0}}{3}\left|1+3 \sin ^{2} \theta_{13}\left(1+2 R_{3}\right)-2 R_{2}\right|
$$

Here the Eq. 21) was used, $R_{2}=\Delta m_{21}^{2} / 4 m_{0}^{2}$ and $R_{3}=\Delta m_{13}^{2} / 4 m_{0}^{2}$. With $m_{0} \approx 0.1 \mathrm{eV}$, the lowest value for the effective neutrino mass may be obtained, then $\left|m_{e e}\right| \geq 0.036 \mathrm{eV}$ for $m_{0} \gtrsim 0.1 \mathrm{eV}$. The predicted regions for $\left|m_{e e}\right|$ can be seen in Fig. 8, in here, let us point out that the parameter space for $m_{0}$ and $|\epsilon|$ is displayed in Figs. 3 and 4 where the $\alpha_{\epsilon}=\pi$ phase was favored according to the analytical study.
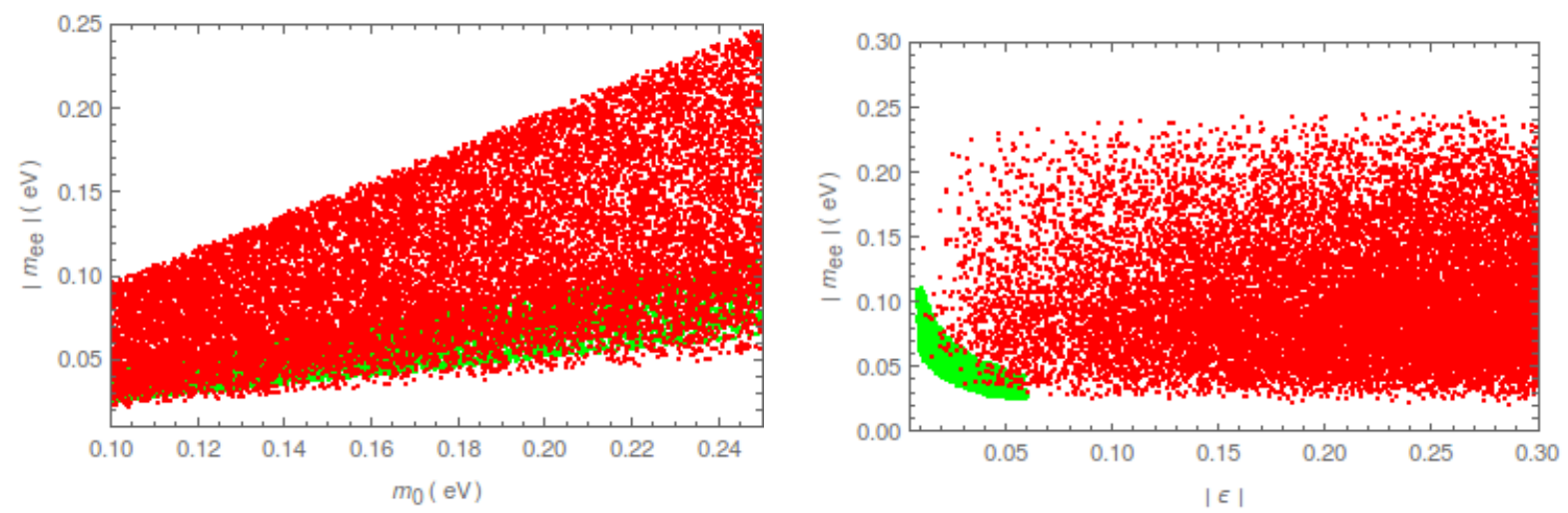

FIG. 8: Allowed region for $\left|m_{e e}\right|$ versus $\left|m_{\nu_{3}}^{0}\right|$ and $|\epsilon|$, respectively.

Let us stress the following, in the case of $\mathrm{CP}$ parities, the breaking of the $\mu-\tau$ symmetry was notable in that very small values for the $|\epsilon|$ parameter were predicted by the model. Including two relative Majorana phases this soft breaking is spoiled as can be seen in Fig. (8). In a similar way to the inverted spectrum, the model predicts small values for $\left|m_{e e}\right|$ in comparison to the GERDA phase-I data, but the allowed region is enhanced by the presence of $\mathrm{CP}$ non trivial values in the Majorana phases. 
Taking into account the $m_{\nu_{e}}$ neutrino mass scale and the sum of the neutrino masses, we have

$$
m_{\nu_{e}} \approx m_{0}\left[1+\frac{2}{3} R_{2}+\frac{1}{2}\left(1+4 R_{3}\right) \sin ^{2} \theta_{13}\right], \quad \sum_{i}\left|m_{\nu_{i}}\right| \approx 3 m_{0}\left[1+\frac{2}{3}\left(R_{2}+R_{3}\right)\right] .
$$

Therefore, we expect that $m_{\nu_{e}} \geq 0.102 \mathrm{eV}$ and $\sum_{i}\left|m_{\nu_{i}}\right| \geq 0.312 \mathrm{eV}$ with $m_{0} \gtrsim 0.1 \mathrm{eV}$. In this ordering, the bound in the neutrino mass scale may be reached if the common neutrino mass is large, at the same time, the sum of the neutrino masses is far away from the bound given by Planck collaboration.
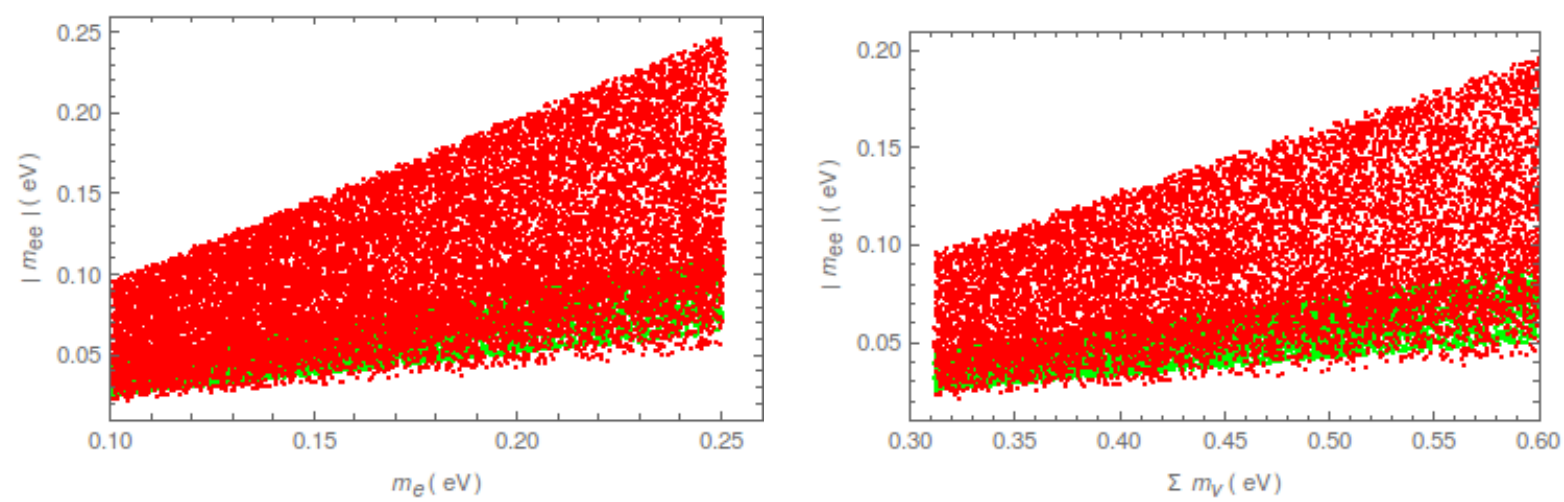

FIG. 9: Predicted region for $\left|m_{e e}\right|$ versus the neutrino mass scale and the sum of neutrino masses.

Similarity to the inverted hierarchy, we add two plots where the atmospheric angle and the effective neutrino mass are displayed against the relative Majorana phases. As we can see, in the first panel, for the atmospheric angle the allowed region has been increased by the presence of CP non trivial values in the Majorana phases; in this framework, two notable regions seem remarkable: a) $\alpha=\pi$ and $\beta=0,2 \pi$; this values correspond to the Case $\mathbf{C} ; b) \alpha, \beta=\pi$, this CP parties correspond to the Case D. These two regions can be distinguished better in the second plot where the allowed values of the effective neutrino mass is shown, in this plot the Case $\mathbf{C}$ seems to be relevant in the presence of $\mathrm{CP}$ non trivial values; in the case of $\mathrm{CP}$ parities, this case was disfavored by predicting large values for the atmospheric angle.
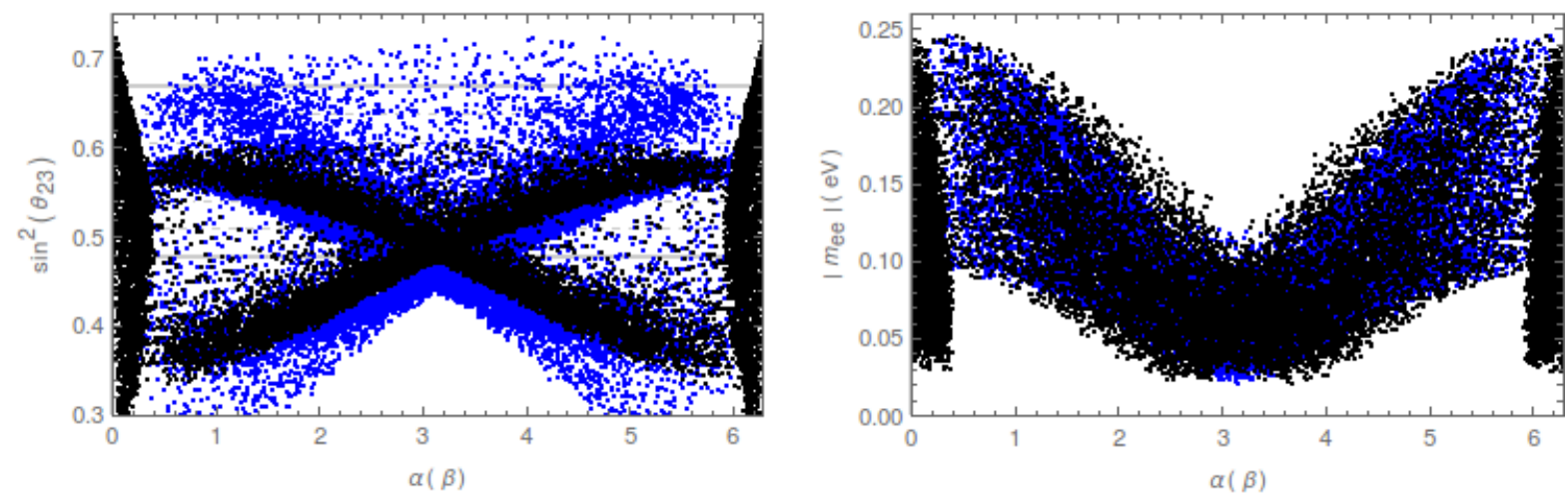

FIG. 10: Blue and black points stand for $\alpha$ and $\beta$ phases, respectively.

As we can notice, the presence of $\mathrm{CP}$ non trivial values for the Majorana phases is a subtle issue since these change the allowed regions for the observables. We ought to comment that an analytical study on the CP non trivial phases should be carried out to verify the naive numerical results. For the moment, this task is out of the scope of this work. 


\section{OUTLOOK AND REMARKS}

We have constructed a $\mathbf{Q}_{6}$ flavored supersymmetric model with non-minimal Higgs sector. In the lepton sector, the $\mu \leftrightarrow \tau$ permutation symmetry is broken only by one perturbative parameter $\epsilon$ which is directly proportional to the inequality $m_{e \tau} \neq m_{e \mu}$. This symmetry breaking deviates the reactor and atmospheric mixing angles from $0^{\circ}$ and $45^{\circ}$, respectively. Such deviations may be sizeable in the presence of CP parities in the Majorana phases, also this particular framework allows us to constrain, in an analytical way, the lightest neutrino mass and the $\epsilon$ parameter that accommodate the deviated mixing angles.

The model predicts that the inverted hierarchy is lesser favored than the degenerate ordering. For this latter mass ordering, the numerical values obtained for the reactor and atmospheric mixing angle are in very good agreement with the current experimental data on neutrino oscillations. Remarkably, the common neutrino mass lies on the favorable region for KamLAND-Zen collaboration and the atmospheric angle lies on the upper octant $\left(\theta_{23}>45^{\circ}\right)$.

Additionally, the effective neutrino mass decay, the neutrino mass scale and the sum of the neutrino mass were calculated in the framework of $\mathrm{CP}$ parities in the Majorana phases. In this particular case, the allowed regions are consistent with the analytical study, however, these regions are enhanced by the contribution of two relative Majorana phases in the neutrino masses. For the effective neutrino mass decay, the allowed values can be reached by the future experiments in this direction.

\section{Acknowledgements}

This work was partially supported by the Mexican grants 237004, PAPIIT IN111115 and Conacyt 132059. JCGI thanks CINVESTAV for the warm hospitality and Red de Altas Energías-CONACYT for the financial support. FGC acknowledges the financial support from CONACYT and PRODEP under Grant No. 511-6/17-8017.

\section{Appendix A: $Q_{6}$ Flavor Symmetry}

The $\mathbf{Q}_{6}$ group has twelve elements which are contained in six conjugacy classes, therefore, it contains six irreducible representations. We will use the notation given in [35, there are various notations and extensive studies for this group, see for example [75, 76]. The $\mathbf{Q}_{6}$ family symmetry has $\mathbf{2}$ two-dimensional irreducible representations denoted

by $\mathbf{2}_{1}$ and $\mathbf{2}_{2}, 4$ one-dimensional ones which are denoted by $\mathbf{1}_{+, 0}, \mathbf{1}_{+, 2}, \mathbf{1}_{-, 1}$ and $\mathbf{1}_{-, 3}$. As it is well known, $\mathbf{2}_{1}$ is a pseudo real and $\mathbf{2}_{2}$ is a real representation. In addition, for $\mathbf{1}_{ \pm, n}$ we have that $n=0,1,2,3$ is the factor $\exp (i n \pi / 2)$ that appears in the matrix given by $\mathbf{B}$. The \pm stands for the change of sign under the transformation given by the $\mathbf{A}$ matrix. So that the first two one-dimensional representations are real and the two latter ones are complex conjugate to each other.

$$
\mathbf{Q}_{6}=\left\{\mathbf{1}, \mathbf{A}, \mathbf{A}^{2}, \mathbf{A}^{3}, \mathbf{A}^{4}, \mathbf{A}^{5}, \mathbf{B}, \mathbf{A B}, \mathbf{A}^{2} \mathbf{B}, \mathbf{A}^{3} \mathbf{B}, \mathbf{A}^{4} \mathbf{B}, \mathbf{A}^{5} \mathbf{B}\right\}
$$

where the $\mathbf{A}$ and $\mathbf{B}$ are two-dimensional matrices whose explicit forms are given by

$$
\mathbf{A}=\left(\begin{array}{cc}
\cos (\pi / 3) & \sin (\pi / 3) \\
-\sin (\pi / 3) & \cos (\pi / 3)
\end{array}\right) \quad \text { and } \quad \mathbf{B}=\left(\begin{array}{cc}
i & 0 \\
0 & -i
\end{array}\right) \text {. }
$$


Let us write the multiplication rules among the six irreducible representations which will be useful to build a phenomenological model:

$$
\begin{aligned}
& \mathbf{1}_{+, 2} \otimes \mathbf{1}_{+, 2}=\mathbf{1}_{+, 0}, \quad \mathbf{1}_{-, 3} \otimes \mathbf{1}_{-, 3}=\mathbf{1}_{+, 2}, \quad \mathbf{1}_{-, 1} \otimes \mathbf{1}_{-, 1}=\mathbf{1}_{+, 2}, \quad \mathbf{1}_{-, 1} \otimes \mathbf{1}_{-, 3}=\mathbf{1}_{+, 0}, \\
& \mathbf{1}_{+, 2} \otimes \mathbf{1}_{-, 1}=\mathbf{1}_{-, 3}, \quad \mathbf{1}_{+, 2} \otimes \mathbf{1}_{-, 3}=\mathbf{1}_{-, 1}, \quad \mathbf{2}_{1} \otimes \mathbf{1}_{+, 2}=\mathbf{2}_{1}, \quad \mathbf{2}_{1} \otimes \mathbf{1}_{-, 3}=\mathbf{2}_{2}, \\
& \mathbf{2}_{1} \otimes \mathbf{1}_{-, 1}=\mathbf{2}_{2}, \quad \mathbf{2}_{2} \otimes \mathbf{1}_{+, 2}=\mathbf{2}_{2}, \quad \mathbf{2}_{2} \otimes \mathbf{1}_{-, 3}=\mathbf{2}_{1}, \quad \mathbf{2}_{2} \otimes \mathbf{1}_{-, 1}=\mathbf{2}_{1} ; \\
& \overbrace{\left(\begin{array}{l}
x_{1} \\
x_{2}
\end{array}\right)}^{\mathbf{2}_{1}} \otimes \overbrace{\left(\begin{array}{l}
y_{1} \\
y_{2}
\end{array}\right)}^{\mathbf{2}_{1}}=\overbrace{\left(x_{1} y_{2}-x_{2} y_{1}\right)}^{\mathbf{1}_{+, 0}}+\overbrace{(\overbrace{\left.x_{1} y_{1}+x_{2} y_{2}\right)}}^{\mathbf{1}_{+, 2}}+\overbrace{\left(\begin{array}{c}
-x_{1} y_{2}-x_{2} y_{1} \\
x_{1} y_{1}-x_{2} y_{2}
\end{array}\right)}^{\mathbf{2}_{2}} \\
& \overbrace{\left(\begin{array}{l}
a_{1} \\
a_{2}
\end{array}\right)}^{\mathbf{2}_{2}} \otimes \overbrace{\left(\begin{array}{c}
b_{1} \\
b_{2}
\end{array}\right)}^{\mathbf{2}_{2}}=\overbrace{\left(a_{1} b_{1}+a_{2} b_{2}\right)}^{\mathbf{1}_{+, 0}}+\overbrace{(\overbrace{\left(a_{1} b_{2}-a_{2} b_{1}\right)}^{\mathbf{1}_{+, 2}}}^{\mathbf{2}_{2}}+\overbrace{\left(\begin{array}{c}
-a_{1} b_{1}+a_{2} b_{2} \\
a_{1} b_{2}+a_{2} b_{1}
\end{array}\right)}^{\mathbf{2}_{2}} \\
& \overbrace{\left(\begin{array}{c}
x_{1} \\
x_{2}
\end{array}\right)}^{\mathbf{2}_{1}} \otimes \overbrace{\left(\begin{array}{l}
a_{1} \\
a_{2}
\end{array}\right)}^{\mathbf{2}_{2}}=\overbrace{\left(x_{1} a_{2}+x_{2} a_{1}\right)}^{\mathbf{1}_{-, 3}}+\overbrace{(\overbrace{\left.x_{1} a_{1}-x_{2} a_{2}\right)}^{\mathbf{1}_{-, 1}}}^{\mathbf{2}_{1}}+\overbrace{\left(\begin{array}{l}
x_{1} a_{1}+x_{2} a_{2} \\
x_{1} a_{2}-x_{2} a_{1}
\end{array}\right)},
\end{aligned}
$$

[1] C. Giunti and C. W. Kim, Fundamentals of Neutrino Physics and Astrophysics, Oxford University Press, USA, illustrated edition edition (2007), ISBN 0198508719,9780198508717,9781429488433.

[2] D. V. Forero, M. Tortola and J. W. F. Valle, Neutrino oscillations refitted, Phys. Rev. D90 (2014) 9093006, arXiv:1405.7540 [hep-ph]

[3] K. Abe et al. (T2K), Observation of Electron Neutrino Appearance in a Muon Neutrino Beam, Phys. Rev. Lett. 112 (2014) 061802, arXiv:1311.4750 [hep-ex]

[4] R. N. Mohapatra and S. Nussinov, Bimaximal neutrino mixing and neutrino mass matrix, Phys.Rev. D60 (1999) 013002 , arXiv:hep-ph/9809415 [hep-ph].

[5] C. Lam, A 2 - 3 symmetry in neutrino oscillations, Phys.Lett. B507 (2001) 214-218, arXiv:hep-ph/0104116 [hep-ph]

[6] T. Kitabayashi and M. Yasue, $S_{2 L}$ permutation symmetry for left-handed $\mu$ and $\tau$ families and neutrino oscillations in an $S U(3)_{L} \times S U(1)_{N}$ gauge model, Phys.Rev. D67 (2003) 015006, arXiv:hep-ph/0209294 [hep-ph]

[7] W. Grimus and L. Lavoura, A Discrete symmetry group for maximal atmospheric neutrino mixing, Phys.Lett.|B572 (2003) 189-195, arXiv:hep-ph/0305046 [hep-ph].

[8] Y. Koide, Universal texture of quark and lepton mass matrices with an extended flavor $2 \leftrightarrow 3$ symmetry, Phys.Rev. D69 (2004) 093001, arXiv:hep-ph/0312207 [hep-ph].

[9] T. Fukuyama and H. Nishiura, Mass matrix of Majorana neutrinos (1997), arXiv:hep-ph/9702253 [hep-ph].

[10] H.-J. He, W. Rodejohann and X.-J. Xu, Origin of Constrained Maximal CP Violation in Flavor Symmetry, Phys. Lett. B751 (2015) 586-594, arXiv:1507.03541 [hep-ph].

[11] Z.-z. Xing and Z.-h. Zhao, A review of $\mu-\tau$ flavor symmetry in neutrino physics, Rept. Prog. Phys. 79 (2016) 7076201. arXiv: 1512.04207 [hep-ph].

[12] C. C. Nishi and B. L. Snchez-Vega, Mu-tau reflection symmetry with a texture-zero, JHEP 01 (2017) 068, arXiv:1611.08282 [hep-ph]

[13] N. Haba and W. Rodejohann, A Supersymmetric contribution to the neutrino mass matrix and breaking of mu-tau symmetry, Phys. Rev. D74 (2006) 017701, arXiv:hep-ph/0603206 [hep-ph]. 
[14] J. C. Gomez-Izquierdo and A. Perez-Lorenzana, Softly broken $\mu \leftrightarrow \tau$ symmetry in the minimal see-saw model, Phys. Rev. D77 (2008) 113015, arXiv:0711.0045 [hep-ph].

[15] D. A. Dicus, S.-F. Ge and W. W. Repko, Generalized Hidden $\mathcal{Z}_{2}$ Symmetry of Neutrino Mixing, Phys. Rev. |D83 (2011) 093007, arXiv: 1012.2571 [hep-ph]

[16] S.-F. Ge, D. A. Dicus and W. W. Repko, $Z_{2}$ Symmetry Prediction for the Leptonic Dirac CP Phase, Phys. Lett. B702 (2011) 220-223, arXiv:1104.0602 [hep-ph]

[17] S.-F. Ge, D. A. Dicus and W. W. Repko, Residual Symmetries for Neutrino Mixing with a Large $\theta_{13}$ and Nearly Maximal $\delta_{D}$, Phys. Rev. Lett. |108 (2012) 041801, arXiv:1108.0964 [hep-ph].

[18] H.-J. He and F.-R. Yin, Common Origin of $\mu-\tau$ and CP Breaking in Neutrino Seesaw, Baryon Asymmetry, and Hidden Flavor Symmetry, Phys. Rev. D84 (2011) 033009, arXiv: 1104.2654 [hep-ph].

[19] T. Araki and Y. Li, $Q_{6}$ flavor symmetry model for the extension of the minimal standard model by three right-handed sterile neutrinos, Phys.Rev. D85 (2012) 065016, arXiv:1112.5819 [hep-ph].

[20] H.-J. He and X.-J. Xu, Octahedral Symmetry with Geometrical Breaking: New Prediction for Neutrino Mixing Angle $\theta_{13}$ and CP Violation, Phys. Rev. D86 (2012) 111301, arXiv:1203.2908 [hep-ph]

[21] A. D. Hanlon, S.-F. Ge and W. W. Repko, Phenomenological consequences of residual $\mathbb{Z}_{2}^{s}$ and $\overline{\mathbb{Z}}_{2}^{s}$ symmetries, Phys. Lett. B729 (2014) 185-191, arXiv:1308.6522 [hep-ph].

[22] S.-F. Ge, Unifying Residual $\mathbb{Z}_{2}^{23} \otimes \mathbb{Z}_{2}^{12}$ Symmetries and Quark-Lepton Complementarity (2014), arXiv:1406.1985 [hep-ph]

[23] S. Luo and Z.-z. Xing, Resolving the octant of $\theta_{23}$ via radiative $\mu-\tau$ symmetry breaking, Phys. Rev. [D90 (2014) 7073005. arXiv:1408.5005 [hep-ph]

[24] D. C. Rivera-Agudelo and A. Prez-Lorenzana, Generating $\theta_{13}$ from sterile neutrinos in $\mu-\tau$ symmetric models, Phys. Rev. D92 (2015) 7 073009, arXiv:1507.07030 [hep-ph]

[25] Z.-z. Xing, H. Zhang and S. Zhou, Nearly Tri-bimaximal Neutrino Mixing and CP Violation from mu-tau Symmetry Breaking, Phys. Lett. B641 (2006) 189-197, arXiv:hep-ph/0607091 [hep-ph].

[26] S.-F. Ge, H.-J. He and F.-R. Yin, Common Origin of Soft mu-tau and CP Breaking in Neutrino Seesaw and the Origin of Matter, JCAP 1005 (2010) 017, arXiv:1001.0940 [hep-ph].

[27] W. Grimus and L. Lavoura, mu-tau Interchange symmetry and lepton mixing, Fortsch. Phys. [61 (2013) 535-545. arXiv:1207.1678 [hep-ph]

[28] Z.-z. Xing and Y.-L. Zhou, A Generic Diagonalization of the $3 \times 3$ Neutrino Mass Matrix and Its Implications on the $\mu-\tau$ Flavor Symmetry and Maximal CP Violation, Phys. Lett. B693 (2010) 584-590, arXiv:1008.4906 [hep-ph].

[29] S. Gupta, A. S. Joshipura and K. M. Patel, How good is $\mu$ - $\tau$ symmetry after results on non-zero $\theta_{13}$ ?, JHEP $\mid 09$ (2013) 035, arXiv: 1301.7130 [hep-ph]

[30] Z.-h. Zhao, On the breaking of mu-tau flavor symmetry, in Conference on New Physics at the Large Hadron Collider Singapore, Singapore, February 29-March 4, 2016 (2016) arXiv:1605.04498 [hep-ph].

[31] P. Chen, G.-J. Ding, F. Gonzalez-Canales and J. W. F. Valle, Generalized $\mu-\tau$ reflection symmetry and leptonic CP violation, Phys. Lett. B753 (2016) 644-652, arXiv:1512.01551 [hep-ph].

[32] P. Chen, G.-J. Ding, F. Gonzalez-Canales and J. W. F. Valle, Classifying CP transformations according to their texture zeros: theory and implications, Phys. Rev.|D94 (2016) 3 033002, arXiv:1604.03510 [hep-ph]

[33] K. S. Babu and J. Kubo, Dihedral families of quarks, leptons and Higgses, Phys.Rev. D71 (2005) 056006 arXiv:hep-ph/0411226 [hep-ph].

[34] Y. Kajiyama, E. Itou and J. Kubo, Nonabelian discrete family symmetry to soften the SUSY flavor problem and to suppress proton decay, Nucl. Phys. B743 (2006) 74-103, arXiv: hep-ph/0511268

[35] Y. Kajiyama, R-parity violation and non-Abelian discrete family symmetry, JHEP 04 (2007) 007, arXiv: hep-ph/0702056

[36] N. Kifune, J. Kubo and A. Lenz, Flavor Changing Neutral Higgs Bosons in a Supersymmetric Extension based on a $Q_{6}$ Family Symmetry, Phys.Rev. D77 (2008) 076010, arXiv:0712.0503 [hep-ph].

[37] K. Babu and Y. Meng, Flavor Violation in Supersymmetric Q6 Model, Phys.Rev. D80 (2009) 075003, arXiv:0907.4231 
[hep-ph]

[38] K. Kawashima, J. Kubo and A. Lenz, Testing the new CP phase in a Supersymmetric Model with Q6 Family Symmetry by $B_{s}$ Mixing, Phys.Lett. B681 (2009) 60-67, arXiv:0907.2302 [hep-ph]

[39] K. Babu, K. Kawashima and J. Kubo, Variations on the Supersymmetric Q6 Model of Flavor, Phys.Rev. D83 (2011) 095008, arXiv: 1103.1664 [hep-ph].

[40] W. Grimus, Realizations of $\mu-\tau$ interchange symmetry, Conf. Proc. C060726 (2006) 312-315, [,312(2006)], arXiv:hep-ph/0610158 [hep-ph].

[41] A. Adulpravitchai, A. Blum and C. Hagedorn, A Supersymmetric D $D_{4}$ Model for $\mu-\tau$ Symmetry, JHEP 03 (2009) 046 arXiv:0812.3799 [hep-ph]

[42] C. Hagedorn and R. Ziegler, $\mu-\tau$ Symmetry and Charged Lepton Mass Hierarchy in a Supersymmetric D $D_{4}$ Model, Phys. Rev. D82 (2010) 053011, arXiv:1007.1888 [hep-ph]

[43] P. F. Harrison and W. G. Scott, Permutation symmetry, tri - bimaximal neutrino mixing and the S3 group characters, Phys. Lett. B557 (2003) 76, arXiv:hep-ph/0302025 [hep-ph].

[44] R. N. Mohapatra, S. Nasri and H.-B. Yu, S3 symmetry and tri-bimaximal mixing, Phys. Lett. B639 (2006) 318-321. arXiv:hep-ph/0605020 [hep-ph].

[45] D. E. Lopez-Fogliani and C. Munoz, Proposal for a Supersymmetric Standard Model, Phys. Rev. Lett. 97 (2006) 041801 , arXiv:hep-ph/0508297 [hep-ph].

[46] J. Fidalgo, D. E. Lopez-Fogliani, C. Munoz and R. Ruiz de Austri, Neutrino Physics and Spontaneous CP Violation in the $\mu \nu S S M$, JHEP 08 (2009) 105, arXiv:0904.3112 [hep-ph].

[47] M. Gell-Mann, P. Ramond and R. Slansky, Complex spinors and unified theories, Conf.Proc. C790927 (1979) 315-321, arXiv:1306.4669 [hep-th]

[48] T. Yanagida, Horizontal gauge symmetry and masses of neutrinos In Proceedings of the Workshop on the Baryon Number of the Universe and Unified Theories, Tsukuba, Japan, 13-14 Feb 1979.

[49] R. N. Mohapatra and G. Senjanovic, Neutrino Masses and Mixings in Gauge Models with Spontaneous Parity Violation, Phys. Rev. D23 (1981) 165

[50] R. N. Mohapatra and G. Senjanovic, Neutrino Mass and Spontaneous Parity Violation, Phys.Rev.Lett. 44 (1980) 912

[51] P. Minkowski, $\mu \rightarrow e \gamma$ at a Rate of One Out of 1-Billion Muon Decays?, Phys. Lett.|B67 (1977) 421.

[52] J. Schechter and J. W. F. Valle, Neutrino Masses in SU(2) ×U(1) Theories, Phys. Rev. D22 (1980) 2227

[53] J. Schechter and J. W. F. Valle, Neutrino Decay and Spontaneous Violation of Lepton Number, Phys. Rev. D25 (1982) 774 .

[54] E. Barradas-Guevara, O. Felix-Beltran, F. Gonzalez-Canales and M. Zeleny-Mora, On the lepton CP violation in a $\nu 2 H D M$ with flavor (2017), arXiv:1704.03474 [hep-ph].

[55] P. Harrison, D. Perkins and W. Scott, Tri-bimaximal mixing and the neutrino oscillation data, Physics Letters B 530 (2002) 1?4 167 - 173, ISSN 0370-2693.

[56] P. Harrison and W. Scott, Symmetries and generalisations of tri-bimaximal neutrino mixing, Physics Letters B 535 (2002) $1 ? 4163-169$, ISSN 0370-2693.

[57] Z. zhong Xing, Nearly tri-bimaximal neutrino mixing and cp violation, Physics Letters B 533 (2002) 1-2 85-93, ISSN 0370-2693.

[58] P. Harrison and W. Scott, Permutation symmetry, tri-bimaximal neutrino mixing and the $s_{3}$ group characters, Physics Letters B $\mid \mathbf{5} 57$ (2003) 1-2 76-86. ISSN 0370-2693.

[59] J. Lesgourgues and S. Pastor, Massive neutrinos and cosmology, Physics Reports |429 (2006) 6 307-379, ISSN 0370-1573.

[60] E. W. Otten and C. Weinheimer, Neutrino mass limit from tritium $\beta$ decay, Reports on Progress in Physics 71 (2008) 8 086201.

[61] F. T. Avignone, S. R. Elliott and J. Engel, Double beta decay, majorana neutrinos, and neutrino mass, Rev. Mod. Phys. 30 (2008) 481-516. 
[62] P. A. R. Ade et al. (Planck), Planck 2015 results. XIII. Cosmological parameters, Astron. Astrophys. 594 (2016) A13, arXiv:1502.01589 [astro-ph.CO]

[63] A. Osipowicz et al. (KATRIN), KATRIN: A Next generation tritium beta decay experiment with sub-eV sensitivity for the electron neutrino mass. Letter of intent (2001), arXiv:hep-ex/0109033 [hep-ex].

[64] O. Host, O. Lahav, F. B. Abdalla and K. Eitel, Forecasting neutrino masses from combining katrin and the cmb observations: Frequentist and bayesian analyses, Phys. Rev. D 76 (2007) 113005.

[65] M. Lindner, A. Merle and W. Rodejohann, Improved limit on $\theta_{13}$ and implications for neutrino masses in neutrinoless double beta decay and cosmology, Phys. Rev. D $\mid 73$ (2006) 053005.

[66] W. Rodejohann, Neutrinoless double beta decay and neutrino physics, J. Phys. G39 (2012) 124008, arXiv:1206.2560 [hep-ph].

[67] S. M. BILENKY and C. GIUNTI, Neutrinoless double-beta decay: A brief review, Modern Physics Letters A 27 (2012) 13 1230015 .

[68] J. Bonn et al., Results from the mainz neutrino mass experiment, Progress in Particle and Nuclear Physics 48 (2002) 1 133 - 139. ISSN 0146-6410.

[69] V. N. Aseev et al., Upper limit on the electron antineutrino mass from the troitsk experiment, Phys. Rev. D 84 (2011) 112003.

[70] J. A. et al., Katrin design report 2004 (available at the website:www.katrin.kit.edu, 245 pages) (2004).

[71] E. W. Otten and C. Weinheimer, Neutrino mass limit from tritium beta decay, Rept. Prog. Phys. 71 (2008) 086201. arXiv:0909.2104 [hep-ex]

[72] F. Capozzi et al., Global constraints on absolute neutrino masses and their ordering, Phys. Rev. D95 (2017) 9096014 , arXiv:1703.04471 [hep-ph].

[73] M. Agostini et al. (GERDA), Results on Neutrinoless Double- $\beta$ Decay of ${ }^{76}$ Ge from Phase I of the GERDA Experiment, Phys. Rev. Lett. 111 (2013) 12 122503, arXiv:1307.4720 [nucl-ex].

[74] M. Agostini et al., Background free search for neutrinoless double beta decay with GERDA Phase II |(2017), [Nature544,47(2017)], arXiv:1703.00570 [nucl-ex].

[75] J. Kubo, Super Flavorsymmetry with Multiple Higgs Doublets, Fortsch.Phys. 61 (2013) 597-621, arXiv:1210.7046 [hep-ph].

[76] H. Ishimori et al., Non-Abelian Discrete Symmetries in Particle Physics, Prog. Theor. Phys. Suppl. 183 (2010) 1-163. arXiv:1003.3552 [hep-th] 